\title{
A generating function for all semi-magic squares and the volume of the Birkhoff polytope
}

\author{
J.A. De Loera $\cdot$ F. Liu $\cdot$ R. Yoshida
}

Received: 2 June 2008 / Accepted: 15 September 2008 / Published online: 9 October 2008

(C) Springer Science+Business Media, LLC 2008

\begin{abstract}
We present a multivariate generating function for all $n \times n$ nonnegative integral matrices with all row and column sums equal to a positive integer $t$, the so called semi-magic squares. As a consequence we obtain formulas for all coefficients of the Ehrhart polynomial of the polytope $B_{n}$ of $n \times n$ doubly-stochastic matrices, also known as the Birkhoff polytope. In particular we derive formulas for the volumes of $B_{n}$ and any of its faces.
\end{abstract}

Keywords Birkhoff polytope · Volume - Lattice points · Generating functions · Ehrhart polynomials

\section{Introduction}

Let $B_{n}$ denote the convex polytope of $n \times n$ doubly-stochastic matrices; that is, the set of real nonnegative matrices with all row and column sums equal to one. The polytope $B_{n}$ is often called the Birkhoff-von Neumann polytope, the assignment polytope, or simply the Birkhoff polytope. It is a well-known problem to compute the volume of $B_{n}$ and there is a fair amount of work on the topic (see [5, 11, 15] and the references therein for information on prior work); in this paper, we present the first exact formula for the volume of $B_{n}$. The formula will follow from a multivariate rational generating function for all possible $n \times n$ integer nonnegative matrices with all row and column sums equal to a positive integer $t$, the so called semi-magic squares [16, 22] (although many authors refer to them as magic squares).

Before stating our main formula, we give a few necessary definitions and notation. We call a directed spanning tree with all edges pointing away from a root $\ell$ an $\ell$ arborescence. The set of all $\ell$-arborescences on the nodes $[n]=\{1,2, \ldots, n\}$ will

J.A. De Loera $(\bowtie) \cdot$ F. Liu $\cdot$ R. Yoshida

University of California Davis, Davis, CA 95616, USA

e-mail: deloera@math.ucdavis.edu 
be denoted by $\operatorname{Arb}(\ell, n)$. It is well known that the cardinality of $\operatorname{Arb}(\ell, n)$ is $n^{n-2}$. For any $T \in \operatorname{Arb}(\ell, n)$, we denote by $E(T)$ the set of directed edges of $T$. As usual let $S_{n}$ be the set of all permutations on [n]. For any $\sigma \in S_{n}$, we associate $\sigma$ with its corresponding permutation matrix, i.e., the $n \times n$ matrix whose $(i, \sigma(i))$ entry is 1 and zero otherwise. Throughout this paper, we will use $\sigma$ to denote both a permutation and the corresponding matrix and it should be clear which one it refers to according to the context. The bracket operator $\langle\cdot, \cdot\rangle$ denotes the dot product of two vectors.

It is well known that given a $d$-dimensional integral polytope $P$, that is a polytope whose vertices have integer coordinates, for any positive integer $t$, the number $e(P, t)$ of lattice points contained in the $t$-th dilation, $t P=\{t X \mid X \in P\}$, is a polynomial of degree $\operatorname{dim}(P)$ in the variable $t$. Furthermore, the leading coefficient of $e(P, t)$ is the normalized volume of $P$ in units equal to the volume of the fundamental domain of the affine lattice spanned by $P$ (see Chapter 4 of [22] or the book [7]). This polynomial is called the Ehrhart polynomial of $P$.

One can find an expression for the Ehrhart polynomial $e\left(B_{n}, t\right)$ of $B_{n}$ using the multivariate generating function

$$
f\left(t B_{n}, \mathbf{z}\right)=\sum_{M \in t B_{n} \cap \mathbb{Z}^{n^{2}}} \mathbf{z}^{M}
$$

of the lattice points of $t B_{n}$, where $\mathbf{z}^{M}=\prod_{1 \leq i, j \leq n} z_{i, j}^{m_{i, j}}$ if $M=\left(m_{i, j}\right)$ is an $n$ by $n$ matrix in $\mathbb{R}^{n^{2}}$. One can see that by plugging $z_{i, j}=1$ for all $i$ and $j$ in $f\left(t B_{n}, \mathbf{z}\right)$, we get $e\left(B_{n}, t\right)$. Our main result is

Theorem 1.1 Given any positive integer $t$, the multivariate generating function for the lattice points of $t B_{n}$ is given by the expression

$$
f\left(t B_{n}, \mathbf{z}\right)=\sum_{\sigma \in S_{n}} \sum_{T \in \operatorname{Arb}(\ell, n)} \mathbf{z}^{t \sigma} \prod_{e \notin E(T)} \frac{1}{\left(1-\prod \mathbf{z}^{W^{T, e} \sigma}\right)},
$$

where $\mathbf{z}^{t \sigma}=\prod_{k=1}^{n} z_{k, \sigma(k)}^{t}$.

Here $W^{T, e}$ denotes the $n \times n(0,-1,1)$-matrix associated to the unique oriented cycle in the graph $T+e$ (see Definition 3.17 for details) and $W^{T, e} \sigma$ denotes the usual matrix multiplication of $W^{T, e}$ and the permutation matrix $\sigma$.

As we apply Lemma 5.4 to Theorem 1.1, we obtain the desired corollary:

Corollary 1.2 For any choice of fixed $\ell \in[n]$, the coefficient of $t^{k}$ in the Ehrhart polynomial $e\left(B_{n}, t\right)$ of the polytope $B_{n}$ of $n \times n$ doubly-stochastic matrices is given by the formula

$$
\frac{1}{k !} \sum_{\sigma \in S_{n}} \sum_{T \in \operatorname{Arb}(\ell, n)} \frac{(\langle c, \sigma\rangle)^{k} \operatorname{td}_{d-k}\left(\left\{\left\langle c, W^{T, e} \sigma\right\rangle, e \notin E(T)\right\}\right)}{\prod_{e \notin E(T)}\left\langle c, W^{T, e} \sigma\right\rangle} .
$$

In the formula $W^{T, e}$ is the $n \times n(0,-1,1)$-matrix associated to the unique oriented cycle in $T+e$ as defined in Definition 3.17 and $W^{T, e} \sigma$ denotes the usual matrix 
multiplication of $W^{T, e}$ and the permutation matrix $\sigma$. The symbol $\operatorname{td}_{j}(S)$ is the $j$-th Todd polynomial evaluated at the numbers in the set $S$ (see Definition 5.1 for details). Finally, $c \in \mathbb{R}^{n^{2}}$ is any vector such that $\left\langle c, W^{T, e} \sigma\right\rangle$ is non-zero for all pairs $(T, e)$ of an $\ell$-arborescence $T$ and a directed edge $e \notin E(T)$ and all $\sigma \in S_{n}$.

As a special case, the normalized volume of $B_{n}$ is given by

$$
\operatorname{vol}\left(B_{n}\right)=\frac{1}{\left((n-1)^{2}\right) !} \sum_{\sigma \in S_{n}} \sum_{T \in \operatorname{Arb}(\ell, n)} \frac{\langle c, \sigma\rangle^{(n-1)^{2}}}{\prod_{e \notin E(T)}\left\langle c, W^{T, e} \sigma\right\rangle} .
$$

We stress that each rational function summand of Formula (1.1) is given only in terms of trees and cycles of a directed complete graph. Our proof of Theorem 1.1 is based on the lattice point rational functions as developed in [4] with some help from the theory of Gröbner bases of toric ideals as outlined in [23].

There is a large collection of prior work on this topic that we mention now to put our result in perspective. In [5] the authors computed the exact value of the volume and the Ehrhart polynomials for up to $n=10$, which is the current record for exact computation. The computations in [5] took several years of computer CPU (running in a parallel machine setup) and our volume formula is so far unable to beat their record without a much more sophisticated implementation. On the other hand, in two recent papers, Canfield and McKay [9, 10] provide simple asymptotic formulas for the volume of $B_{n}$ as well as the number of lattice points of $t B_{n}$. However, our closed formula for the volume of $B_{n}$ is nonetheless interesting for the following reasons. First, as it was demonstrated in [1, 12], the faces of $B_{n}$ are also quite interesting for combinatorics and applications. For example all network polytopes appear as faces of a large enough $B_{n}$. From our formula it is easy to work out volume formulas for any concrete face of $B_{n}$. We demonstrate this possibility in the case of the well-known $C R Y_{n}$ polytope [12] whose volume is equal to the product of the first $n-1$ Catalan numbers (see [25]). Concretely, we obtain for the first time the Ehrhart polynomials of facets of $B_{n}$ and $C R Y_{n}$ for $n \leq 7$. In principle, this could be applied to derive formulas for the number of integral flows on networks. Second, not only we can derive formulas for the coefficients of the Ehrhart polynomial of $B_{n}$, but we can also derive formulas for the integral of any polynomial function over $B_{n}$. We hope our generating function will be useful for various problems over the set of all semi-magic squares, at least for small values of $n$.

This paper is organized as follows: In Section 2 we begin with background material that will be used in the later sections, including background properties of $B_{n}$, a short discussion of Gröbner bases and triangulations, Brion's theorem and generating functions for lattice points in polyhedra. In that section, we sketch the steps we will follow to compute the generating function of lattice points inside cones. In Section 3 we discuss the triangulations of the dual cone at each vertex of $B_{n}$ which we encode via Gröbner bases. From Brion's formula we derive in Section 4 a sum of rational functions encoding all the lattice points of the dilation $t B_{n}$ and thus a proof of Theorem 1.1. In Section 5 we show how from Theorem 1.1 we can derive all the coefficients of the Ehrhart polynomial of $B_{n}$ after expressing the generating function in terms of Todd polynomials. Finally, in Section 6, we explain how to obtain Ehrhart polynomials and formulas of integration for any face of $B_{n}$. 


\section{Background}

For basic definitions about convex polytopes which are not stated in this paper, please see [26]. Chapters 5 and 6 in [24] have a very detailed introduction to $B_{n}$ and transportation polytopes. For all the details and proofs about lattice point counting and their multivariate generating functions see $[3,4,7]$. We begin with some useful facts about the polytope $B_{n}$. It is well known that the vertices of $B_{n}$ are precisely the $n \times n$ permutation matrices. Permutation matrices are in bijection with matchings on the complete bipartite graph $K_{n, n}$. The polytope $B_{n}$ lies in the $n^{2}$-dimensional real space $\mathbb{R}^{n^{2}}=\{n \times n$ real matrices $\}$, and we use $M(i, j)$ to denote the $(i, j)$-entry of a matrix $M$ in the space. There is a graph theoretic description of the edges of $B_{n}$; they correspond to the cycles in $K_{n, n}$. On the other hand, for each pair $(i, j)$ with $1 \leq i, j \leq n$, the set of doubly-stochastic matrices with $(i, j)$ entry equal to 0 is a facet (a maximal proper face) of $B_{n}$ and all facets arise in this way. It is also easy to see that the dimension of $B_{n}$ is $(n-1)^{2}$ (i.e., the volume we wish to compute is the $(n-1)^{2}$-volume of $B_{n}$ regarded as a subset of $n^{2}$-dimensional Euclidean space). Note that an $n \times n$ doubly-stochastic matrix is uniquely determined by its upper left $(n-1) \times(n-1)$ submatrix. The set of $(n-1) \times(n-1)$ matrices obtained this way is the set $A_{n}$ of all nonnegative $(n-1) \times(n-1)$ matrices with row and column sums $\leq 1$ such that the sum of all the entries is at least $n-2$. $A_{n}$ is affinely isomorphic to $B_{n}$ and we often compute in $A_{n}$ instead of $B_{n}$ because $A_{n}$ is full-dimensional.

Cones and Generating functions for lattice points For any polytope $P \in \mathbb{R}^{d}$, we would like to write a generating function for the following sum encoding the lattice points of $P$

$$
\sum_{\alpha \in P \cap \mathbb{Z}^{d}} \mathbf{z}^{\alpha}
$$

where $\mathbf{z}^{\alpha}=z_{1}^{\alpha_{1}} z_{2}^{\alpha_{2}} \cdots z_{d}^{\alpha_{d}}$. We give now a step-by-step description of how the generating function is constructed.

A cone is the set of all linear nonnegative combinations of a finite set of vectors. If a cone contains no other linear subspace besides the origin then we say it is pointed. Given a cone $C \subset \mathbb{R}^{d}$, the dual cone to $C$ is a cone $C^{*}=\left\{y \in \mathbb{R}^{d} \mid\langle x, y\rangle \geq 0, \forall x \in\right.$ $C\}$. The following lemma states some properties of dual cones. (See Theorem 9.1 in [21] for a proof).

Lemma 2.1 Let $C$ be a pointed cone in $\mathbb{R}^{n}$, and let $D=C^{*}$ be its dual cone. Then the following properties hold

(1) $C$ is the dual cone of $D$, namely $C=D^{*}=\left(C^{*}\right)^{*}$.

(2) If $C$ is a full dimensional pointed cone, then so is D. Moreover, if $\left\{F_{i}\right\}$ is the set of facets of $C$, then $D$ is precisely the cone generated by the set of rays $\left\{R_{i}\right\}$ satisfying, for any $i$,

$R_{i}$ is perpendicular to $F_{i}$, and for any ray $R$ of $C$ not on $F_{i}:\left\langle R, R_{i}\right\rangle>0$. 
Now if $P$ is a polytope and $v$ is a vertex of $P$, the supporting polyhedron of $P$ at $v$ is

$$
S(P, v)=v+\left\{u \in \mathbb{R}^{d}: v+\delta u \in P \text { for all sufficiently small } \delta>0\right\},
$$

and the supporting cone of $P$ at $v$ is defined as $C(P, v)=S(P, v)-v$.

For a set $A \subset \mathbb{R}^{d}$, the indicator function $[A]: \mathbb{R}^{d} \rightarrow \mathbb{R}$ of $A$ is defined as

$$
[A](x)= \begin{cases}1 & \text { if } x \in A, \\ 0 & \text { if } x \notin A .\end{cases}
$$

The algebra of polyhedra $\mathbb{P}\left(\mathbb{R}^{d}\right)$ is the vector space over $\mathbb{Q}$ spanned by the indicator functions $[P]$ of all polyhedra $P \subset \mathbb{R}^{d}$. The algebra of polytopes $\mathbb{P}_{P}\left(\mathbb{R}^{d}\right)$ is the subspace spanned by the indicator functions of the polytopes in $\mathbb{R}^{d}$. The algebra of cones $\mathbb{P}_{C}\left(\mathbb{R}^{d}\right)$ is the subspace spanned by the indicator functions of the polyhedral cones in $\mathbb{R}^{d}$. A linear transformation

$$
\Phi: \mathbb{P}\left(\mathbb{R}^{d}\right) \rightarrow V
$$

where $V$ is a vector space over $\mathbb{Q}$ is called a valuation. Similarly, linear transformations defined on $\mathbb{P}_{P}\left(\mathbb{R}^{d}\right)$ and $\mathbb{P}_{C}\left(\mathbb{R}^{d}\right)$ are also called valuations [4].

One important tool for counting lattice points is the ability of expressing the indicator function of a simplicial cone as an integer linear combination of the indicator functions of unimodular simplicial cones. Given a cone $K \subset \mathbb{R}^{d}$, we say that the finite family of cones $K_{i}, i \in I=\{1,2, \ldots, l\}$ is a decomposition of $K$ if there are numbers $\epsilon_{i} \in\{-1,1\}$ such that

$$
[K]=\sum_{i \in I} \epsilon_{i}\left[K_{i}\right]
$$

Theorem 2.2 (Theorem 3.1 and its proof in [4]) There is a map $\mathfrak{F}$ which, to each rational polyhedron $P \subset \mathbb{R}^{d}$, associates a unique rational function $f(P, \mathbf{z})$ in $d$ complex variables $\mathbf{z} \in \mathbb{C}^{d}, \mathbf{z}=\left(z_{1}, \ldots, z_{d}\right)$, such that the following properties are satisfied:

(i) The map $\mathfrak{F}$ is a valuation.

(ii) If $P$ is pointed, there exists a nonempty open subset $U_{p} \subset \mathbb{C}^{d}$, such that $\sum_{\alpha \in P \cap \mathbb{Z}^{d}} \mathbf{z}^{\alpha}$ converges absolutely to $f(P, \mathbf{z})$ for all $\mathbf{z} \in U_{P}$.

(iii) If $P$ is pointed, then $f(P, \mathbf{z})$ satisfies

$$
f(P, \mathbf{z})=\sum_{\alpha \in P \cap \mathbb{Z}^{d}} \mathbf{z}^{\alpha}
$$

for any $\mathbf{z} \in \mathbb{C}^{d}$ where the series converges absolutely.

(iv) If $P$ is not pointed, i.e., $P$ contains a line, then $f(P, \mathbf{z})=0$.

Because the rational function $f(P, \mathbf{z})$ encodes the lattice points of $P$, we call $f(P, \mathbf{z})$ the multivariate generating function of the lattice points (MGF) of $P$. The rational function has an expression as a sum of simple terms, but to describe them we need the following facts. 
Theorem 2.3 (Brion, 1988; Lawrence, 1991, see [4, 6] for proofs) Let $P$ be a rational polyhedron and let $V(P)$ be the vertex set of $P$. Then,

$$
f(P, \mathbf{z})=\sum_{v \in V(P)} f(S(P, v), \mathbf{z}) .
$$

This theorem reduces the problem of finding the MGF of a rational polyhedron $P$ to that of finding the MGF of the supporting polyhedra at each vertex of $P$. If the vertex of the supporting polyhedron is integral we can simply assume the vertex is the origin and work instead with supporting cones.

Corollary 2.4 If $P$ an integral polyhedron, i.e., all the vertices of $P$ are integral vertices, then

$$
f(P, \mathbf{z})=\sum_{v \in V(P)} \mathbf{z}^{v} f(C(P, v), \mathbf{z})
$$

Although it is in general more complicated to give the MGF of an arbitrary cone, if the cone is unimodular, its MGF has a simple form:

Lemma 2.5 (Lemma 4.1 in [4]) If $K$ is a d-dimensional pointed cone in $\mathbb{R}^{n}$ generated by the rays $\left\{r_{i}\right\}_{1 \leq i \leq d}$, where the $r_{i}$ 's form a $\mathbb{Z}$-basis of the lattice $\mathbb{Z}^{n} \cap \operatorname{span}(K)$ $(\operatorname{span}(K)$ is the $d$-subspace where $K$ lies), then we say $K$ is a unimodular cone and we have that

$$
f(K, \mathbf{z})=\prod_{i=1}^{d} \frac{1}{1-\mathbf{z}^{r_{i}}} .
$$

Barvinok gave an algorithm to decompose any pointed cone $C$ as a signed sum of simple unimodular cones [4] and thus deriving an expression for $f(P, \mathbf{z})$ as a sum of terms like those in Lemma 2.5. In principle, one needs to keep track of lower dimensional cones in the decomposition for writing an inclusion-exclusion formula of the MGF $f(C, \mathbf{z})$. Fortunately, by using the Brion's polarization trick (see Remark 4.3 in [4]), one only needs to consider full-dimensional cones. This trick involves using dual cones of a decomposition of the dual cone to $C$ instead of directly decomposing $C$. The main idea is to note that the duals of low dimensional cones are not pointed and thus, from Part (iv) of Theorem 2.2, their associated rational functions vanish.

Now we are ready to sketch the main steps of Barvinok's algorithm to compute $f(C, \mathbf{z})$ (see [4] for details):

\section{Algorithm}

Input: a rational full-dimensional pointed cone $C$.

Output: the MGF of $C: f(C, \mathbf{z})$.

(1) Find the dual cone $D=C^{*}$ to $C$.

(2) Apply the Barvinok decomposition to $D$ into a set of unimodular cones $D_{i}$ which have the same dimension as $D$ (ignoring all the lower dimensional cones).

(3) Find the dual cone $C_{i}$ to each $D_{i}$. The cone $C_{i}$ will be unimodular as well. 
(4) $f(C, \mathbf{z})=\sum_{i} \epsilon_{i} f\left(C_{i}, \mathbf{z}\right)$, where $\epsilon_{i}$ is +1 or -1 determined by Barvinok decomposition.

This algorithm is still not right for us; the algorithm is for full-dimensional cones, however, the cones we need to study are not full-dimensional since the Birkhoff polytope is not full-dimensional. Also, Lemma 2.1 provides us a way to compute the rays of $C^{*}$ if $C$ is full-dimensional and pointed. Hence, it will be nice if we can make our cones full-dimensional. What we will do is properly project cones into a lower dimensional space so that they become full-dimensional.

Definition 2.6 Let $V \subset \mathbb{R}^{n}$ and $W \subset \mathbb{R}^{m}$ be vector spaces with full rank lattices $L_{V}:=V \cap \mathbb{Z}^{n}$ and $L_{W}:=W \cap \mathbb{Z}^{m}$, respectively. A linear map $\phi$ from $V$ to $W$ is a good projection if $\phi$ gives a bijection between $L_{V}$ and $L_{W}$. Note that because of the linearity of $\phi$, the lattices $L_{V}$ and $L_{W}$ have the same rank.

Lemma 2.7 Suppose $V, W$ are as in Definition 2.6 and $\phi$ is a good projection from $V$ to $W$. Because $\phi$ is a linear map, we can consider $\phi$ as given by a certain $m \times n$ matrix $\phi=\left(\phi_{i, j}\right)$. We define a map $\Phi: \mathbb{C}^{m} \rightarrow \mathbb{C}^{n}$ by mapping $\mathbf{y}=\left(y_{1}, \ldots, y_{m}\right) \in \mathbb{C}^{m}$ to $\mathbf{z}=\left(z_{1}, \ldots, z_{n}\right) \in \mathbb{C}^{n}$, where

$$
z_{j}=\prod_{i=1}^{m} y_{i}^{\phi_{i, j}}
$$

Then the following statements hold.

(1) $\operatorname{dim}(V)=\operatorname{dim}(W)$.

(2) $\phi$ gives an isomorphism between $V$ and $W$ that preserves the lattice. Therefore, there exists an inverse (linear) map $\phi^{-1}$ from $W$ to $V$ that preserves the lattice as well. Thus, $\phi^{-1}$ is also a good projection from $W$ to $V$.

(3) $C$ is a unimodular cone in $V$ if and only if $\phi(C)$ is a unimodular cone in $W$.

(4) For any $\alpha \in \mathbb{Z}^{n}$ and $\mathbf{y} \in \mathbb{C}^{m}$, if $\beta=\phi(\alpha)$ and $\mathbf{z}=\Phi(\mathbf{y})$, then $\mathbf{y}^{\beta}=\mathbf{z}^{\alpha}$.

(5) For any pointed rational polyhedron $P \in V$, the series $\sum_{\beta \in \phi(P) \cap \mathbb{Z}^{m}} \mathbf{y}^{\beta}$ converges absolutely if and only if the series $\sum_{\alpha \in P \cap \mathbb{Z}^{n}} \Phi(\mathbf{y})^{\alpha}$ converges absolutely. Furthermore, we have

$$
f(\phi(P), \mathbf{y})=f(P, \Phi(\mathbf{y})) .
$$

(6) Let $P_{1}, P_{2}, \ldots, P_{k}$ be pointed rational polyhedra in $V$, and $a_{1}, \ldots, a_{k} \in \mathbb{C}$, then

$$
f(P, \mathbf{z})=\sum a_{i} f\left(P_{i}, \mathbf{z}\right) \Leftrightarrow f(\phi(P), \mathbf{y})=\sum a_{i} f\left(\phi\left(P_{i}\right), \mathbf{y}\right) .
$$

Proof The proofs of (1), (2) and (3) follow from the fact that good projections give lattices of same rank and thus isomorphic vector spaces. For the proof of (4), $\beta=$ $\phi(\alpha)$ implies that $\beta_{i}=\sum_{j=1}^{n} \phi_{i, j} \alpha_{j}$. Thus,

$$
\mathbf{z}^{\alpha}=\prod_{j=1}^{n} z_{j}^{\alpha_{j}}=\prod_{j=1}^{n} \prod_{i=1}^{m} y_{i}^{\phi_{i, j} \alpha_{j}}=\prod_{i=1}^{m} \prod_{j=1}^{n} y_{i}^{\phi_{i, j} \alpha_{j}}=\prod_{i=1}^{m} y_{i}^{\sum_{j=1}^{n} \phi_{i, j} \alpha_{j}}=\prod_{i=1}^{m} y_{i}^{\beta_{i}}=\mathbf{y}^{\beta} .
$$


Because $\phi$ is a good projection, the lattice in $P$ and the lattice in $\phi(P)$ are in oneto-one correspondence under $\phi$. Therefore, to prove (4), it is enough to show that if $\beta=\phi(\alpha)$ and $\mathbf{z}=\Phi(\mathbf{y})$, then $\mathbf{y}^{\beta}=\mathbf{z}^{\alpha} . \beta=\phi(\alpha)$ implies that $\beta_{i}=\sum_{j=1}^{n} \phi_{i, j} \alpha_{j}$. Thus,

$$
\mathbf{z}^{\alpha}=\prod_{j=1}^{n} z_{j}^{\alpha_{j}}=\prod_{j=1}^{n} \prod_{i=1}^{m} y_{i}^{\phi_{i, j} \alpha_{j}}=\prod_{i=1}^{m} \prod_{j=1}^{n} y_{i}^{\phi_{i, j} \alpha_{j}}=\prod_{i=1}^{m} y_{i}^{\sum_{j=1}^{n} \phi_{i, j} \alpha_{j}}=\prod_{i=1}^{m} y_{i}^{\beta_{i}}=\mathbf{y}^{\beta} .
$$

The first part of (5) follows immediately from (4). Let $Y$ be the set of $\mathbf{y} \in \mathbb{C}^{m}$ for which the series $\sum_{\beta \in \phi(P) \cap \mathbb{Z}^{m}} \mathbf{y}^{\beta}$ converges absolutely and $Z$ be the set of $\mathbf{z} \in$

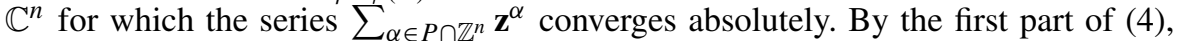
$\Phi(Y) \subset Z$. By Theorem 2.2, $f(P, \mathbf{z})=\sum_{\alpha \in P \cap \mathbb{Z}^{n}} \mathbf{z}^{\alpha}$ for any $\mathbf{z} \in Z$. In particular, $f(P, \mathbf{z})=\sum_{\alpha \in P \cap \mathbb{Z}^{n}} \mathbf{z}^{\alpha}$ for any $\mathbf{z} \in \Phi(Y)$. Hence, for any $\mathbf{y} \in Y$.

$$
f(P, \Phi(\mathbf{y}))=\sum_{\alpha \in P \cap \mathbb{Z}^{n}} \Phi(y)^{\alpha}=\sum_{\beta \in \phi(P) \cap \mathbb{Z}^{m}} \mathbf{y}^{\beta} .
$$

We use Theorem 2.2 again to conclude that $f(P, \Phi(y))$ is the rational function $f(\phi(P), \mathbf{y})$ associated to $\phi(P)$.

Given (2), we only need to check one direction in (6). Suppose $f(P, \mathbf{z})=$ $\sum a_{i} f\left(P_{i}, \mathbf{z}\right)$. We can apply (2.2) to both sides to obtain $f(\phi(P), \mathbf{y})=$ $\sum a_{i} f\left(\phi\left(P_{i}\right), \mathbf{y}\right)$.

Using Lemma 2.7, we modify Barvinok's algorithm and sketch a method to construct $f(C, \mathbf{z})$ for supporting cones $C$ at vertices of $B_{n}$. We will try to follow this sequence of steps in Section 3:

(CMGF) Method for constructing the multivariate generating function for lattice points of a cone

Input: a rational (not necessarily full-dimensional) pointed cone $C \subset \mathbb{R}^{n}$.

Output: the MGF of $C: f(C, \mathbf{z})$.

(0) Let $V$ be the subspace spanned by $C$ in $\mathbb{R}^{n}$. Find a subspace $W$ of $\mathbb{R}^{m}$ together with a good projection $\phi$ from $V$ to $W$. Let $\bar{C}=\phi(C)$.

(1) Find a dual cone $\bar{D}$ to $\bar{C}$.

(2) Decompose $\bar{D}$ into addition and subtraction of unimodular cones $\bar{D}_{i}$ which have the same dimension as $\bar{D}$, ignoring all the lower dimensional cones.

(3) Find dual cone $\bar{C}_{i}$ of each $\overline{D_{i}}$. Note, that $\overline{C_{i}}$ is also unimodular. Let $C_{i}=$ $\phi^{-1}\left(\bar{C}_{i}\right)$.

(4) $f(C, \mathbf{z})=\sum_{i} \epsilon_{i} f\left(C_{i}, \mathbf{z}\right)$, where $\epsilon_{i}$ is +1 or -1 determined by the signed decomposition.

In the next section, we will apply the method (CMGF) step-by-step to the supporting cone at the vertex $I$, the identity permutation. We will get the MGF of this supporting cone and, by applying the action of the symmetric group $S_{n}$, we can deduce the MGF of all other supporting cones of vertices of $B_{n}$ and thus, by Theorem 2.3, the MGF of $B_{n}$. We will see later, in Section 5 , that the knowledge of $f(P, \mathbf{z})$ as a sum of rational functions yields a rational function formula for the volume of $P$. 
Triangulations and Gröbner bases of toric ideals For step (2) in our step-by-step construction of the generating function, we will show (Lemma 3.4) that in fact any triangulation of the dual cone of the supporting cone of a vertex already gives a set of unimodular cones (hence, the $\epsilon_{i}$ 's in Step (4) are all +1 ). A triangulation of a cone $C$ is a special decomposition of a cone as the union of simplicial cones with disjoint interiors whose union covers completely the cone $C$. In this article we use polynomial ideals to codify the triangulations, namely toric ideals and their Gröbner bases. See Chapter 8 in [23] for all details. Here are the essential notions:

Fix a set $\mathcal{A}=\left\{a_{1}, a_{2}, \ldots, a_{n}\right\} \subset \mathbb{Z}^{d}$. For any $\mathbf{u}=\left(u_{1}, u_{2}, \ldots, u_{n}\right) \in \mathbb{Z}^{n}$, we let

$$
\mathbf{u} \mathcal{A}:=u_{1} a_{1}+u_{2} a_{2}+\cdots+u_{n} a_{n} .
$$

For any $\mathbf{u} \in \mathbb{Z}^{d}$, we denote by $\operatorname{supp}(\mathbf{u}):=\left\{i \mid u_{i} \neq 0\right\}$ the support of $\mathbf{u}$. Every $\mathbf{u} \in \mathbb{Z}^{d}$ can be written uniquely as $\mathbf{u}=\mathbf{u}^{+}-\mathbf{u}^{-}$, where $\mathbf{u}^{+}$and $\mathbf{u}^{-}$are nonnegative and have disjoint support.

Definition 2.8 The toric ideal of $\mathcal{A}, I_{\mathcal{A}} \subset k[\mathbf{x}]:=k\left[x_{1}, x_{2}, \ldots, x_{n}\right]$ is the ideal generated by the binomials

$$
I_{\mathcal{A}}:=\left\langle\mathbf{x}^{\mathbf{u}^{+}}-\mathbf{x}^{\mathbf{u}^{-}} \mid \mathbf{u} \mathcal{A}=0\right\rangle .
$$

Given a real vector $\lambda=\left(\lambda_{1}, \ldots, \lambda_{n}\right)$ in $\mathbb{R}^{n}$, we can define a monomial order $>_{\lambda}$ such that for any $a, b \in \mathbb{Z}_{>0}^{n}$, their monomials satisfy $\mathbf{x}^{a}>_{\lambda} \mathbf{x}^{b}$ if $\langle a, \lambda\rangle>\langle b, \lambda\rangle$ and ties are broken via the lexicographic order. Using the ordering of monomials we can select the initial monomial of a polynomial $f$ with respect to $>_{\lambda}$, i.e., the highest term present. We will denote it by $i n_{>_{\lambda}}(f)$. For an ideal $I$ contained in $\mathbb{C}\left[x_{1}, . ., x_{n}\right]$ its initial ideal is the ideal $i n_{>_{\lambda}}(I)$ generated by the initial monomials of all polynomials in $I$. A finite subset of polynomials $G=\left\{g_{1}, \ldots, g_{n}\right\}$ of an ideal $I$ is a Gröbner basis of $I$ with respect to $>_{\lambda}$ if $i n_{>_{\lambda}}(I)$ is generated by $\left\{i n_{>_{\lambda}}\left(g_{1}\right), \ldots, i n_{>_{\lambda}}\left(g_{n}\right)\right\}$. In other words, $G$ is a Gröbner basis for $I$ if the initial monomial of any polynomial in $I$ is divisible by one of the monomials $i n_{>\lambda}\left(g_{i}\right)$. It can be proved from the definition that a Gröbner basis is a generating set for the ideal $I$. As we will state later, each Gröbner basis of the toric ideal $I_{\mathcal{A}}$ yields a regular triangulation of the convex hull of $\mathcal{A}$. The fact that triangulations constructed using Gröbner bases are regular will not be used in our construction.

A subdivision of $\mathcal{A}$ is a collection $T$ of subsets of $\mathcal{A}$, called cells, whose convex hulls form a polyhedral complex with support $Q=\operatorname{conv}(\mathcal{A})$. If each cell in $T$ is a simplex, then $T$ is called a triangulation of $\mathcal{A}$. Every vector $\lambda=\left(\lambda_{1}, \ldots, \lambda_{n}\right)$ in $\mathbb{R}^{n}$ induces a subdivision of $\mathcal{A}=\left\{a_{1}, \ldots, a_{n}\right\}$ as follows. Consider the polytope $Q_{\lambda}=\operatorname{conv}\left(\left\{\left(a_{1}, \lambda_{1}\right), \ldots,\left(a_{n}, \lambda_{n}\right)\right\}\right)$ which lies in $\mathbb{R}^{d+1}$. Generally, $Q_{\lambda}$ is a polytope of dimension $\operatorname{dim}(\operatorname{conv}(\mathcal{A}))+1$. The lower envelope of $Q_{\lambda}$ is the collection of faces of the form $\left\{x \in Q_{\lambda} \mid\langle c, x\rangle=c_{0}\right\}$ with $Q_{\lambda}$ contained in the halfspace $\langle c, x\rangle \leq c_{0}$ where the last coordinate $c_{d+1}$ is negative. The lower envelope of $Q_{\lambda}$ is a polyhedral complex of dimension $\operatorname{dim}(\operatorname{conv}(\mathcal{A}))$. We define $T_{\lambda}$ as the subdivision of $\mathcal{A}$ whose cells are the projections of the cells of the lower envelope of $Q_{\lambda}$. In other words, $\left\{a_{i_{1}}, a_{i_{2}}, \ldots, a_{i_{k}}\right\}$ is a cell of $T_{\lambda}$ if $\left\{\left(a_{i_{1}}, \lambda_{i_{1}}\right),\left(a_{i_{2}}, \lambda_{i_{2}}\right), \ldots,\left(a_{i_{k}}, \lambda_{i_{k}}\right)\right\}$ are the vertices 
of a face in the lower envelope of $Q_{\lambda}$. The subdivision $T_{\lambda}$ is called a regular subdivision of $\mathcal{A}$. We remark that just as a triangulation can be uniquely specified by its maximal dimensional simplices, it can also be uniquely expressed by its minimal non-faces (minimal under containment). Now we are ready to state the algebratriangulation correspondence:

Theorem 2.9 (See proof in Chapter 8 of [23]) Let $\mathcal{A}$ be an $n \times d$ matrix with integer entries, whose row vectors $\left\{a_{1}, \ldots, a_{n}\right\}$ span an affine space of dimension $d-1$. Let $I_{\mathcal{A}}$ be the toric ideal defined by $\mathcal{A}$. Then, the minimal non-faces of the regular triangulation of $\mathcal{A}$ associated to the vector $\lambda$ can be read from the generators of the radical of the initial ideal of the Gröbner basis of $I_{\mathcal{A}}$ with respect to the term order $>_{\lambda}$. More precisely, for $\lambda$ generic, the radical of the initial ideal of $I_{\mathcal{A}}$ equals

$$
\left\langle x_{i_{1}} x_{i_{2}} \cdots x_{i_{s}}:\left\{i_{1}, i_{2}, \ldots, i_{s}\right\} \text { is a minimal non-face of } T_{\lambda}\right\rangle=\bigcap_{\sigma \in T_{\lambda}}\left\langle x_{i}: i \notin \sigma\right\rangle \text {. }
$$

The crucial fact we will use is that the maximal simplices of the regular triangulation $T_{\lambda}$ are transversals to the supports of the monomials from the initial ideal of the Gröbner basis. In the next section, we will apply Theorem 2.9 to create a triangulation of the dual cones.

To the readers who are unfamiliar with commutative algebra language, using a Gröbner basis to describe a triangulation may not feel totally necessary or clear. Thus, we explain here the advantages of doing it this way. First, traditionally checking that a set of simplices is a triangulation of $\mathcal{A}$ is not trivial since one has to verify they have disjoint interiors (which requires a full description of all linear dependences of the rays) and that the union of the simplicial cones fully covers the convex hull of $\mathcal{A}$. But, having a Gröbner basis avoids checking these two tedious geometric facts. Second, the initial monomials of the Gröbner bases are precisely the minimal nonfaces of the triangulation $T_{\lambda}$, which are complementary to the maximal simplicial cones of the triangulation. From the point of view of efficiency, the encoding of a simplicial complex via its non-faces is sometimes much more economic than via its maximal facets. For more on the theory of triangulations see [14].

\section{The MGF of the supporting cone of $B_{n}$ at the vertex $I$}

Due to the transitive action of the symmetric group on $B_{n}$ it is enough to explain a method to compute the MGF of the supporting cone at the vertex associated to the identity permutation (we denote this by $I$ ) and then simply permute the results. Nevertheless it is important to stress that, although useful and economical, there is no reason to use the same triangulation at each vertex. Similarly, the triangulations we use are all regular, but for our purposes there is no need for this property either.

There are $n^{2}$ facets of $B_{n}$ : for any fixed $(i, j): 1 \leq i, j \leq n$, the collection of permutation matrices $P$ satisfying $P(i, j)=0$ defines a facet $F_{i, j}$ of $B_{n}$. Hence, every permutation matrix is on exactly $n(n-1)$ facets and the vertex $I$ is on the facets $F_{i, j}, i \neq j$. Let $C_{n}$ be the supporting cone at the identity matrix $I$, then the set of 
facets of $C_{n}$ is $\left\{F_{i, j}-I\right\}_{1 \leq i, j \leq n, i \neq j}$. (Note that we need to subtract the vertex $I$ from $F_{i, j}$ because the supporting cone is obtained by shifting the supporting polyhedron at the vertex $I$ to the origin.) We are going to apply our method CMGF to find the MGF of $C_{n}$.

\subsection{Step 0: A good projection}

$C_{n}$, as well $B_{n}$, lie in the $n^{2}$-dimensional space $\mathbb{R}^{n^{2}}=\{n \times n$ real matrices $\}$. But they lie in different affine subspaces (the vertex of $C_{n}$ is the origin). Let $V_{n}$ be the subspace of $\mathbb{R}^{n^{2}}$ spanned by $C_{n}$. It is easy to see that

$$
V_{n}=\left\{M \in \mathbb{R}^{n^{2}} \mid \sum_{k=1}^{n} M(i, k)=\sum_{k=1}^{n} M(k, j)=0, \forall i, j\right\} .
$$

Let $W_{n}$ be the vector space $\mathbb{R}^{(n-1)^{2}}=\{(n-1) \times(n-1)$ real matrices $\}$. We define a linear map $\phi$ from $\mathbb{R}^{n^{2}}$ to $W_{n}$ by ignoring the entries in the last column and the last row of a matrix in $\mathbb{R}^{n^{2}}$, that is, for any $M$, we define $\phi(M)$ to be the matrix $(M(i, j))_{1 \leq i, j \leq n-1}$. One can check that the restriction $\phi$ to $V_{n}$ is a good projection from $V_{n}$ to $W_{n}$. Let

$$
\bar{C}_{n}:=\phi\left(C_{n}\right) .
$$

Also, let $\bar{F}_{i, j}=\phi\left(F_{i, j}\right)$ and $\bar{P}=\phi(P)$, for any permutation matrix $P$ on $[n]$. (These are actually the facets and vertices of $A_{n}$, which is the full-dimensional version of $B_{n}$, as we explained at the beginning of Section 2.) By the linearity of $\phi$ the facets of $\bar{C}_{n}$ are $\left\{\bar{F}_{i, j}-\bar{I}\right\}_{1 \leq i, j \leq n, i \neq j}$, and $\bar{F}_{i, j}$ is defined by the collection of $\bar{P}$ 's where $P$ 's are permutation matrices (on $[n]$ ) satisfying $P(i, j)=0$.

3.2 Step 1: The dual cone $\bar{D}_{n}$ to $\bar{C}_{n}$

The cone $\bar{C}_{n}$ is full dimensional in $W=\mathbb{R}^{(n-1)^{2}}$. Hence, we can use Lemma 2.1 to find its dual cone. We will first define a cone, and then show it is the dual cone to $\bar{C}_{n}$.

Definition 3.1 $\bar{D}_{n}$ is the cone spanned by rays $\left\{\bar{M}_{i, j}\right\}_{1 \leq i, j \leq n, i \neq j}$, where $\bar{M}_{i, j}$ is the $(n-1)$ by $(n-1)$ matrix such that

(i) the $(i, j)$-entry is 1 and all other entries equal zero, if $i \neq n$ and $j \neq n$;

(ii) the entries on the ith row are all -1 and all other entries equal zero, if $i \neq n$ and $j=n$;

(iii) the entries on the $j$ th column are all -1 and all other entries equal zero, if $i=n$ and $j \neq n$. 
Example 3.2 (Example of $\bar{M}_{i, j}$ when $n=3$ ) Here we present each 2 by 2 matrix $\bar{M}_{i, j}$ as a row vector, which is just the first and second row of the matrix listed in this order.

$\begin{array}{lrrrr}\bar{M}_{1,3}: & -1 & -1 & 0 & 0 \\ \bar{M}_{2,3}: & 0 & 0 & -1 & -1 \\ \bar{M}_{3,1}: & -1 & 0 & -1 & 0 \\ \bar{M}_{3,2}: & 0 & -1 & 0 & -1 \\ \bar{M}_{1,2}: & 0 & 1 & 0 & 0 \\ \bar{M}_{2,1}: & 0 & 0 & 1 & 0\end{array}$

Lemma 3.3 $\bar{D}_{n}$ is the dual cone to $\bar{C}_{n}$ inside the vector space $W_{n}$.

Proof For any $i, j \in[n]$ and $i \neq j$, we need to check that condition (2.1) is satisfied. Note that a ray of $\bar{C}_{n}$ is given by the vector $\bar{P}-\bar{I}$, for $P$ a permutation matrix adjacent to the identity permutation. Thus it is enough to show that for any permutation matrix $P$ on $[n]$, we have $\left\langle\bar{M}_{i, j}, \bar{P}\right\rangle \geq\left\langle\bar{M}_{i, j}, \bar{I}\right\rangle$ and the equality holds if and only if $\bar{P}$ is on $\bar{F}_{i, j}$, or equivalently, $P$ is on the facet $F_{i, j}$. We have the following three situations for verification:

(i) If $i \neq n$ and $j \neq n,\left\langle\bar{M}_{i, j}, \bar{P}\right\rangle$ is 0 if $P$ is on $F_{i, j}$ and is 1 if $P$ is not on $F_{i, j}$.

(ii) If $i \neq n$ and $j=n,\left\langle\bar{M}_{i, j}, \bar{P}\right\rangle$ is -1 if $P$ is on $F_{i, j}$ and is 0 if $P$ is not on $F_{i, j}$.

(iii) If $i=n$ and $j \neq n$, it is the same as (ii).

Therefore, $\bar{D}_{n}$ is the dual cone to $\bar{C}_{n}$.

\subsection{Step 2: The triangulations of $\bar{D}_{n}$}

As we mentioned in the last section, we will use the idea of toric ideal to find a triangulation of the dual cone $\bar{D}_{n}$ to decompose $\bar{D}_{n}$ into unimodular cones.

Lemma 3.4 Let $\mathcal{M}$ be the configuration of vectors $\left\{\bar{M}_{i, j}\right\}_{1 \leq i, j \leq n, i \neq j}$ and $[\mathcal{M}]$ denote the matrix associated to $\mathcal{M}$, i.e, the rows of $[\mathcal{M}]$ are the vectors in $\mathcal{M}$ written as row vectors. The matrix $[\mathcal{M}]$ is totally unimodular, i.e., for any $(n-1)^{2}$ linearly independent $\bar{M}_{i, j}$ 's, they span a unimodular cone. It follows that all triangulations of the cone $\bar{D}_{n}$ have the same number of maximal dimensional simplices.

Proof Up to a rearrangement of rows the matrix [M] will look as follows: The first few rows are the negatives of the vertex-edge incidence matrix of the complete bipartite $K_{n-1, n-1}$, then under those rows we have $n-1$ cyclically arranged copies of an $(n-2) \times(n-2)$ identity matrix. It is well known that the vertex-edge incidence matrix of the complete bipartite $K_{n-1, n-1}$ is totally unimodular. Moreover it is also known, see e.g., Theorem 19.3 in [21], that a matrix $A$ is totally unimodular if each collection of columns of $A$ can be split into two parts so that the sum of the columns in one part minus the sum of the columns in the other part is a vector with entries $0,+1$, and -1 . This characterization of totally unimodular matrices is easy to verify in our matrix $[\mathcal{M}]$ because whatever partition that works for the column sets of the 
vertex-edge incidence matrix of the complete bipartite $K_{n-1, n-1}$ works also for the corresponding columns of $\mathcal{M}$, because of the diagonal structure of the rows below it.

The fact that all triangulations have the same number of maximal simplices follows from the unimodularity as proved in Corollary 8.9 of [23].

Therefore, any triangulation of $\bar{D}_{n}$ gives a decomposition of $\bar{D}_{n}$ into a set of unimodular cones. Since $\mathcal{M}$ defines the vertex figure of $\bar{D}_{n}$, it is sufficient to triangulate the convex hull of $\mathcal{M}$. Hence, we consider the toric ideal

$$
I_{\mathcal{M}}:=\left\langle\mathbf{x}^{\mathbf{u}^{+}}-\mathbf{x}^{\mathbf{u}^{-}} \mid \mathbf{u} \mathcal{M}=0\right\rangle
$$

of $\mathcal{M}$ inside the polynomial ring $k[\mathbf{x}]:=k\left[x_{i, j}: 1 \leq i, j \leq n, i \neq j\right]$. Note that here $\mathbf{u} \in \mathbb{Z}^{n(n-1)}$ is an $n(n-1)$ dimensional vector indexed by $\{(i, j): i, j \in[n], i \neq j\}$.

Recall that a circuit of $I_{\mathcal{A}}$ is an irreducible binomial $\mathbf{x}^{\mathbf{u}^{+}}-\mathbf{x}^{\mathbf{u}^{-}}$in $I_{\mathcal{A}}$ which has minimal support. Another result follows immediately from Lemma 3.3, Lemma 3.4 and [23, Proposition 4.11,Proposition 8.11]:

Lemma 3.5 The set $\mathcal{C}_{\mathcal{M}}$ of circuits of the homogeneous toric ideal $I_{\mathcal{M}}$ is in fact a universal Gröbner basis $\mathcal{U}_{\mathcal{M}}$ for $I_{\mathcal{M}}$.

For any partition of $[n]=S \cup T$, we denote by $\mathbf{u}_{S, T} \in \mathbb{Z}^{n(n-1)}$ the $n(n-1)$ dimensional vector, where

$$
\mathbf{u}_{S, T}(i, j)= \begin{cases}1, & \text { if } i \in S, j \in T \\ -1, & \text { if } i \in T, j \in S, \\ 0, & \text { otherwise }\end{cases}
$$

One can easily check that $\mathbf{u}_{S, T}$ has the following two properties:

$$
\mathbf{u}_{S, T}(i, j)+\mathbf{u}_{S, T}(j, i)=0, \text { for any } i \neq j .
$$

$$
\mathbf{u}_{S, T}(i, j)+\mathbf{u}_{S, T}(j, k)+\mathbf{u}_{S, T}(k, i)=0 \text {, for any distinct } i, j \text { and } k .
$$

We define

$$
P_{S, T}:=\mathbf{x}^{\mathbf{u}_{S, T}^{+}}-\mathbf{x}^{\mathbf{u}_{S, T}^{-}}=\prod_{s \in S, t \in T} x_{s, t}-\prod_{s \in S, t \in T} x_{t, s}
$$

where $\mathbf{u}_{S, T}^{+}(i, j)=\left\{\begin{array}{ll}1, & \text { if } i \in S, j \in T, \\ 0, & \text { otherwise, }\end{array}\right.$ and $\mathbf{u}_{S, T}^{-}(i, j)= \begin{cases}1, & \text { if } i \in T, j \in S, \\ 0, & \text { otherwise }\end{cases}$

Proposition 3.6 The set of circuits of $I_{\mathcal{M}}$ consists of all the binomials $P_{S, T}$ 's:

$$
\mathcal{C}_{\mathcal{M}}=\left\{P_{S, T} \mid S \cup T \text { is a partition of }[n]\right\} .
$$


Example 3.7 For $n=3$, we have

$$
\begin{aligned}
\mathcal{C}_{\mathcal{M}}=\left\{P_{\{1\},\{2,3\}}\right. & =x_{1,2} x_{1,3}-x_{2,1} x_{3,1}, \\
& P_{\{2,3\},\{1\}}=x_{2,1} x_{3,1}-x_{1,2} x_{1,3}, \\
& P_{\{2\},\{1,3\}}=x_{2,1} x_{2,3}-x_{1,2} x_{3,2}, \\
& P_{\{1,3\},\{2\}}=x_{1,2} x_{3,2}-x_{2,1} x_{2,3}, \\
& P_{\{3\},\{1,2\}}=x_{3,1} x_{3,2}-x_{1,3} x_{2,3}, \\
& \left.P_{\{1,2\},\{3\}}=x_{1,3} x_{2,3}-x_{3,1} x_{3,2}\right\} .
\end{aligned}
$$

We break the proof of Proposition 3.6 into several lemmas. Before we state and prove the lemmas, we give a formula for the entries in

$$
\mathbf{u} \mathcal{M}=\sum_{i, j \in[n], i \neq j} \mathbf{u}(i, j) \bar{M}_{i, j}
$$

For any $i, j \in[n-1]$, at most three members of $\mathcal{M}$ are nonzero at $(i, j)$-entry: $\bar{M}_{i, j}(i, j)=1$ (this one does not exist if $i=j$ ), $\bar{M}_{i, n}(i, n)=-1$, and $\bar{M}_{n, j}(n, j)=$ -1 . Hence,

$$
(\mathbf{u} \mathcal{M})(i, j)= \begin{cases}-\mathbf{u}(i, n)-\mathbf{u}(n, j) & i=j \\ \mathbf{u}(i, j)-\mathbf{u}(i, n)-\mathbf{u}(n, j) & i \neq j\end{cases}
$$

Therefore, we have the following lemma.

\section{Lemma 3.8}

$$
\mathbf{u} \mathcal{M}=0 \text { if and only if } \begin{cases}\mathbf{u}(i, n)+\mathbf{u}(n, i)=0, & \forall i \in[n-1] \\ \mathbf{u}(i, j)-\mathbf{u}(i, n)-\mathbf{u}(n, j)=0, & \forall i \neq j \in[n-1]\end{cases}
$$

Lemma 3.9 For any partition of $[n]=S \cup T$, we have that $\mathbf{u}_{S, T} \mathcal{M}=0$. Hence $P_{S, T}$ is in the toric ideal $I_{\mathcal{M}}$.

Proof It directly follows from (3.2), (3.3), and Lemma 3.8.

Lemma 3.10 For any nonzero $\mathbf{u} \in \mathbb{Z}^{n(n-1)}$ satisfying $\mathbf{u} \mathcal{M}=0$, i.e., $\mathbf{x}^{\mathbf{u}^{+}}-\mathbf{x}^{\mathbf{u}^{-}} \in I_{\mathcal{M}}$, there exists a partition of $[n]=S \cup T$, so that $\operatorname{supp}\left(\mathbf{u}_{S, T}\right) \subset \operatorname{supp}(\mathbf{u})$.

Proof We first show that there exists $t \in[n]$, such that either $(t, n)$ or $(n, t)$ is in the $\operatorname{support} \operatorname{supp}(\mathbf{u})$ of $\mathbf{u}$. Let $(i, j) \in \operatorname{supp}(\mathbf{u})$, if either $i$ or $j$ is $n$, then we are done. Otherwise, by Lemma 3.8, we must have either $(i, n)$ or $(n, j)$ in $\operatorname{supp}(\mathbf{u})$.

By Lemma 3.8 again, we conclude that $(t, n) \in \operatorname{supp}(\mathbf{u})$ if and only if $(n, t) \in$ $\operatorname{supp}(\mathbf{u})$. Let $T=\{t \mid(t, n) \in \operatorname{supp}(\mathbf{u})$ and/or $(n, t) \in \operatorname{supp}(\mathbf{u})\}$ and $S=[n] \backslash T$. Both $S$ and $T$ are nonempty. Thus $S \cup T$ is a partition of $[n]$. We will show that $S \cup T$ is the partition needed to finish the proof. 
$\operatorname{supp}\left(\mathbf{u}_{S, T}\right)=\{(s, t) \mid s \in S, t \in T\} \cup\{(t, s) \mid s \in S, t \in T\}$. Hence, we need to show that $\forall s \in S, \forall t \in T$, both $(s, t)$ and $(t, s)$ are in $\operatorname{supp}(\mathbf{u})$. If $s=n$, it follows immediately from the definition of $T$. If $s \neq n,(s, n) \notin \operatorname{supp}(\mathbf{u})$ since $s \notin T$. Therefore, $(\mathbf{u} \mathcal{M})(s, t)=\mathbf{u}(s, t)-\mathbf{u}(n, t)$, which implies that $(s, t) \in \operatorname{supp}(\mathbf{u})$. We can similarly show that $(t, s) \in \operatorname{supp}(\mathbf{u})$ as well.

Lemma 3.11 Let $\mathbf{u} \in \mathbb{Z}^{n(n-1)}$ be satisfying $\mathbf{u} \mathcal{M}=0$, and $\operatorname{supp}(\mathbf{u})=\operatorname{supp}\left(\mathbf{u}_{S, T}\right)$ for some partition of $[n]=S \cup T$, then $\exists c \in \mathbb{Z}$ such that $\mathbf{u}=c \mathbf{u}_{S, T}$.

Proof Because $\mathbf{u}_{S, T}=-\mathbf{u}_{T, S}$, we can assume that $n \in S$. Fix $t_{0} \in T$, and let $c:=$ $\mathbf{u}\left(n, t_{0}\right)$, we will show that $\mathbf{u}=c \mathbf{u}_{S, T}$. Basically, we need to show that $\forall s \in S$ and $\forall t \in T, \mathbf{u}(s, t)=\mathbf{u}\left(n, t_{0}\right)$ and $\mathbf{u}(t, s)=-\mathbf{u}\left(n, t_{0}\right)$. We will show it case by case, by using Lemma 3.8 and the fact that $\mathbf{u}(s, n)=\mathbf{u}(n, s)=0$ when $s \neq n$ and $\mathbf{u}\left(t_{0}, t\right)=0$ when $t \neq t_{0}$.

- If $s=n, t=t_{0}: \mathbf{u}(s, t)=\mathbf{u}\left(n, t_{0}\right)$ and $\mathbf{u}(t, s)=\mathbf{u}\left(t_{0}, n\right)=-\mathbf{u}\left(n, t_{0}\right)$.

- If $s=n, t \neq t_{0}: \mathbf{u}(s, t)=\mathbf{u}(n, t)=\mathbf{u}\left(t_{0}, t\right)-\mathbf{u}\left(t_{0}, n\right)=\mathbf{u}\left(n, t_{0}\right)$ and $\mathbf{u}(t, s)=$ $\mathbf{u}(t, n)=-\mathbf{u}(n, t)=-\mathbf{u}\left(n, t_{0}\right)$.

- If $s \neq n, t=t_{0}: \mathbf{u}(s, t)=\mathbf{u}\left(s, t_{0}\right)=\mathbf{u}(s, n)+\mathbf{u}\left(n, t_{0}\right)=\mathbf{u}\left(n, t_{0}\right)$ and $\mathbf{u}(t, s)=$ $\mathbf{u}\left(t_{0}, s\right)=\mathbf{u}\left(t_{0}, n\right)+\mathbf{u}(n, s)=-\mathbf{u}\left(n, t_{0}\right)$.

- If $s \neq n, t \neq t_{0}: \mathbf{u}(s, t)=\mathbf{u}(s, n)+\mathbf{u}(n, t)=\mathbf{u}\left(n, t_{0}\right)$ and $\mathbf{u}(t, s)=\mathbf{u}(t, n)+$ $\mathbf{u}(n, s)=-\mathbf{u}\left(n, t_{0}\right)$.

Proof of Proposition 3.6 By Lemma 3.9, Lemma 3.10 and Lemma 3.11, we know that

$$
\mathcal{C}_{\mathcal{M}} \subset\left\{P_{S, T} \mid S \cup T \text { is a partition of }[n]\right\} .
$$

Now we only need to show that for any partition $S \cup T$, there does not exist another partition $S^{\prime} \cup T^{\prime}$ such that $\operatorname{supp}\left(\mathbf{u}_{S^{\prime}, T^{\prime}}\right)$ is strictly contained in $\operatorname{supp}\left(\mathbf{u}_{S, T}\right)$. Suppose we have two such partitions and let $(i, j) \in \operatorname{supp}\left(\mathbf{u}_{S, T}\right) \backslash \operatorname{supp}\left(\mathbf{u}_{S^{\prime}, T^{\prime}}\right)$. Then $i$ and $j$ are both in $S^{\prime}$ or $T^{\prime}$. Without loss of generality, we assume they are both in $S^{\prime}$. Let $t \in T^{\prime}$, then $(i, t)$ and $(j, t)$ are both in the support of $\mathbf{u}_{S^{\prime}, T^{\prime}}$, thus in the support of $\mathbf{u}_{S, T}$. But the fact that $(i, j) \in \operatorname{supp}(S, T)$ indicates that one of $i$ and $j$ is in $S$ and the other one is in $T$. Wherever $t$ is in, we cannot have both $(i, t)$ and $(j, t)$ in the support of $\mathbf{u}_{S, T}$. Therefore, we proved that each $P_{S, T}$ is a circuit.

Corollary 3.12 For any $\ell \in[n]$,

$$
G r_{\ell}:=\left\{P_{S, T} \mid S \cup T \text { is a partition of }[n] \text { s.t. } \ell \in S\right\}
$$

is a Gröbner basis of $\mathcal{M}$ with respect to any term order $<$ satisfying $x_{\ell, j}>x_{i, k}$, for any $i \neq \ell$. Thus, the set of initial monomials of the elements in $G r_{\ell}$ are

$$
\operatorname{Ini}\left(G r_{\ell}\right):=\left\{\prod_{s \in S, t \in T} x_{s, t} \mid S \cup T \text { is a partition of }[n] \text { s.t. } \ell \in S\right\} \text {. }
$$


Example 3.13 For $n=3, \ell=3$ :

$$
\begin{aligned}
G r_{\ell}=\{ & P_{\{2,3\},\{1\}}=x_{2,1} x_{3,1}-x_{1,2} x_{1,3}, \\
& P_{\{1,3\},\{2\}}=x_{1,2} x_{3,2}-x_{2,1} x_{2,3}, \\
& \left.P_{\{3\},\{1,2\}}=x_{3,1} x_{3,2}-x_{1,3} x_{2,3}\right\}
\end{aligned}
$$

and

$$
\operatorname{Ini}\left(G r_{\ell}\right)=\left\{x_{2,1} x_{3,1}, x_{1,2} x_{3,2}, x_{3,1} x_{3,2}\right\}
$$

Recall that $\operatorname{Arb}(\ell, n)$ is the set of all $\ell$-arborescences on $[n]$. For any $T \in$ $\operatorname{Arb}(\ell, n)$, we define the support of $T$ to be $\operatorname{supp}(T):=\{(i, j) \mid i$ is the parent of $j$ in $T\}$, and let $\mathcal{M}(T)=\left\{\bar{M}_{i, j} \mid(i, j) \in \operatorname{supp}(T)\right\}$ be the corresponding subset of $\mathcal{M}$ defined in Lemma 3.4. (Note that the support of $T$ is actually the same as the edge set $E(T)$ of $T$. We call it support here to be consistent with the definitions of other supports.)

Proposition 3.14 For any arborescence $T$ on $[n]$, we define $\bar{D}_{T}$ to be the cone generated by the rays in the set $\mathcal{M} \backslash \mathcal{M}(T)$, i.e., $D_{T}=\operatorname{cone}(\mathcal{M} \backslash \mathcal{M}(T))$.

Fix any $\ell \in[n]$. The term order and Gröbner basis described in Corollary 3.12 give us a triangulation of $\bar{D}_{n}$ :

$$
\operatorname{Tri}_{\ell}:=\left\{\bar{D}_{T} \mid T \in \mathbf{A r b}(\ell, n)\right\}
$$

Proof From the theory of Gröbner bases of toric ideals in Theorem 2.9, the maximal simplices are given by the set of transversals, all minimal sets $\sigma \subset\{(i, j) \mid i \neq j \in$ $[n]\}$ such that $\sigma \cap \operatorname{supp}(m) \neq \emptyset, \forall m \in \operatorname{Ini}\left(G r_{\ell}\right)$. Now due to the fact that each of the initial monomials are in bijection to the cuts of the complete graph, the transversals are indeed given by all possible arborescences

$$
\{\operatorname{supp}(T) \mid T \in \operatorname{Arb}(\ell, n)\} .
$$

One direction is easy: given any arborescence $T$ on $[n]$ with root $\ell$, one sees that $\operatorname{supp}(T)$ is a transversal. We show the other direction: given a transversal $\sigma$, we can draw a directed graph $G_{\sigma}$ according to $\sigma$, i.e., $\operatorname{supp}\left(G_{\sigma}\right)=\sigma$. We let $T$ be the set of all $i$ 's such that there does not exist a directed path from $\ell$ to $i$. $T$ is empty, because otherwise $m=\prod_{s \notin T, t \in T} x_{s, t} \in \operatorname{Ini}\left(G r_{\ell}\right)$ but $\sigma \cap \operatorname{supp}(m)=\emptyset$. Therefore, for any vertex $i$, there exists a directed path from $\ell$ to $i$. This implies that there is an $\ell$ arborescence as a subgraph of $G_{\sigma}$. However, by the minimality of $\sigma, G_{\sigma}$ has to be this arborescence.

Finally, from Theorem 2.9 we know that the complement of these transversals is precisely the set of simplices of the triangulation. 

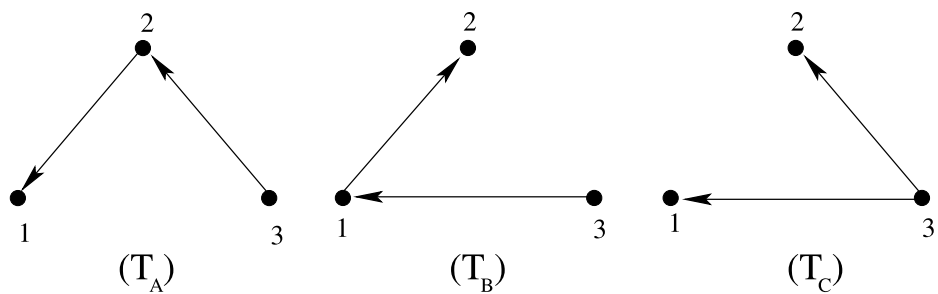

Fig. 1 3-arborescences

Example 3.15 For $n=3, \ell=3$, there are only three trees for $K_{3}$, thus the three 3-arborescences $T_{A}, T_{B}, T_{C}$ for $K_{3}$ are depicted in Figure 1.

$$
\begin{aligned}
\operatorname{Tri}_{\ell}=\left\{\bar{D}_{T_{A}}\right. & =\operatorname{cone}\left(\mathcal{M} \backslash \mathcal{M}\left(T_{A}\right)\right)=\operatorname{cone}\left(\left\{\bar{M}_{1,3}, \bar{M}_{2,3}, \bar{M}_{3,1}, \bar{M}_{1,2}\right\}\right) \\
& \bar{D}_{T_{B}}=\operatorname{cone}\left(\mathcal{M} \backslash \mathcal{M}\left(T_{B}\right)\right)=\operatorname{cone}\left(\left\{\bar{M}_{1,3}, \bar{M}_{2,3}, \bar{M}_{3,2}, \bar{M}_{2,1}\right\}\right) \\
\bar{D}_{T_{C}} & \left.=\operatorname{cone}\left(\mathcal{M} \backslash \mathcal{M}\left(T_{C}\right)\right)=\operatorname{cone}\left(\left\{\bar{M}_{1,3}, \bar{M}_{2,3}, \bar{M}_{1,2}, \bar{M}_{2,1}\right\}\right)\right\},
\end{aligned}
$$

where $\bar{M}_{i, j}$ is defined as in Example 3.2.

3.4 Step 3: The dual cone to $\bar{D}_{T}$

We have given triangulations $\operatorname{Tr}_{\ell}$ of $\bar{D}_{n}$. By Lemma 3.4, we know this gives a decomposition of $\bar{D}_{n}$ into a set of unimodular cones $\bar{D}_{T}$, one for each arborescence $T$. Hence we can proceed to find the dual cone to each $\bar{D}_{T}$ inside $W_{n}$.

Recall that $V_{n}$ is the subspace spanned by the supporting cone $C_{n}$ at the vertex $I$ and can be described by (3.1). We will define cone $C_{T}$, for each $T \in \operatorname{Arb}(\ell, n)$, in the subspace $V_{n}$, then show that $\bar{C}_{T}:=\phi\left(C_{T}\right)$ is the dual cone to $\bar{D}_{T}$.

Definition 3.16 For any directed edge $e=(s, t)$ ( $s$ is pointed to $t$ ) we define the weight of $e$ to be the $n \times n$ matrix $w(e) \in \mathbb{R}^{n^{2}}$, whose $(s, t)$-entry is $1,(t, t)$-entry is -1 , and all the remaining entries are zeros.

Given $T$ an arborescence on $[n]$ with root $\ell$, let $v$ be a vertex of $T$. Then there is a unique path from $\ell$ to $v$. We define the weight $w^{T}(v)$ of $v$ with respect to $T$ to be the summation of the weights of all the edges on this path.

Definition 3.17 Let $T$ be an arborescence on $[n]$ with root $\ell$. For each directed edge $e=(i, j)$ not in $T$, i.e., $e \notin E(T)$, we define

$$
W^{T, e}:=w^{T}(s)-w^{T}(t)+w(e)
$$


More precisely, the entries of $W^{T, e}$ are

$$
W^{T, e}(i, j)= \begin{cases}1, & \begin{array}{l}
\text { if } i \neq j \text { and }(i, j) \in \operatorname{cycle}(T+e) \text { has the same } \\
\text { orientation as } e,
\end{array} \\
-1, & \begin{array}{l}
\text { if } i \neq j \text { and }(i, j) \in \operatorname{cycle}(T+e) \text { has the opposite } \\
\text { orientation as } e,
\end{array} \\
-1, & \begin{array}{l}
\text { if } i=j \text { and } i \text { is a vertex in two edges of cycle }(T+e) \text { with } \\
\text { both edges having same orientation as } e .,
\end{array} \\
1, & \begin{array}{l}
\text { if } i=j \text { and } i \text { is a vertex in two edges of cycle }(T+e), \text { with } \\
\text { both edges having opposite orientation of e., }
\end{array} \\
0, & \text { in all other cases. }\end{cases}
$$

where cycle $(T+e)$ denote the unique cycle created by adding e to $T$.

Let $C_{T}$ be the cone generated by the set of rays $\left\{W^{T, e} \mid e \notin E(T)\right\}$ and $\bar{C}_{T}$ be its projection under $\phi$ (the map that ignores the last row and last column of an $n \times n$ matrix):

$$
C_{T}:=\operatorname{cone}\left(\left\{W^{T, e} \mid e \notin E(T)\right\}\right), \bar{C}_{T}:=\phi\left(C_{T}\right) .
$$

\section{Proposition 3.18}

(1) Each $W^{T, e}$ is in the subspace

$$
V_{n}=\left\{M \in \mathbb{R}^{n^{2}} \mid \sum_{k=1}^{n} M(i, k)=\sum_{k=1}^{n} M(k, j)=0, \forall i, j\right\} .
$$

Hence, $C_{T}$ is in $V_{n}$.

(2) $\bar{C}_{T}$ is the dual cone to $\bar{D}_{T}$ in the vector space $W_{n}=\mathbb{R}^{(n-1)^{2}}$.

\section{Proof}

(1) We observe that for each row or column of $W^{T, e}$, there are either one 1 , one -1 and the other entries are zeros or all entries are zeros.

(2) $\bar{C}_{T}$ is the cone generated by the set of rays $\left\{\phi\left(W^{T, e}\right) \mid e \notin E(T)\right\}$, and $\bar{D}_{T}$ is the cone generated by the set of rays $\left\{\bar{M}_{i, j} \mid(i, j) \notin \operatorname{supp}(T)\right\}$. Recall $\phi$ is the map that ignores the entries in the last column and the last row of a matrix in $V_{n} \subset \mathbb{R}^{n^{2}}$. Hence, we have

$$
\phi\left(W^{T, e}\right)(k, \ell)=W^{T, e}(k, \ell), \forall 1 \leq k, \ell \leq n-1 .
$$

To check whether $\bar{C}_{T}$ is the dual cone to $\bar{D}_{T}$, it is enough to check for any directed edge $e=(s, t) \notin E(T)$ and any $(i, j) \notin \operatorname{supp}(T)$, we have $\left\langle\phi\left(W^{T, e}\right), \bar{M}_{i, j}\right\rangle$ is positive when $(i, j)=(s, t)$ and is 0 otherwise. In fact, we will show that $\left\langle\phi\left(W^{T, e}\right), \bar{M}_{i, j}\right\rangle=\delta_{(i, j),(s, t)}$. There are three situations.

- If $i \neq n$ and $j \neq n$, then $\left\langle\phi\left(W^{T, e}\right), \bar{M}_{i, j}\right\rangle=\phi\left(W^{T, e}\right)(i, j)=W^{T, e}(i, j)$.

- If $i=n$ and $j \neq n$, then $\left\langle\phi\left(W^{T, e}\right), \bar{M}_{i, j}\right\rangle=\sum_{k=1}^{n-1}\left(-\phi\left(W^{T, e}\right)(k, j)\right)=$ $\sum_{k=1}^{n-1}\left(-W^{T, e}(k, j)\right)=W^{T, e}(n, j)=W^{T, e}(i, j)$. 
- If $i \neq n$ and $j=n$, similarly we have $\left\langle\phi\left(W^{T, e}\right), \bar{M}_{i, j}\right\rangle=W^{T, e}(i, j)$.

Hence, for every situation $\left\langle\phi\left(W^{T, e}\right), \bar{M}_{i, j}\right\rangle=W^{T, e}(i, j)$. However, since the only edge in cycle $(T+e)$ not in $T$ is $e, W^{T, e}(i, j)=\delta_{(i, j),(s, t)}$.

Example 3.19 When $n=3, \ell=3$, as before we will present $W^{T, e}$ as a row vector, which is just the first, second and last row of the matrix in order. For the 3arborescence $T_{A}$ in Figure 1, we have four directed edges to be added, the edges $(1,2),(1,3),(3,1)$ and $(2,3)$.

$\begin{array}{lccccccccc}W^{T_{A},(1,2)}: & -1 & 1 & 0 & 1 & -1 & 0 & 0 & 0 & 0 \\ W^{T_{A},(1,3)}: & -1 & 0 & 1 & 1 & -1 & 0 & 0 & 1 & -1 \\ W^{T_{A},(2,3)}: & 0 & 0 & 0 & 0 & -1 & 1 & 0 & 1 & -1 \\ W^{T_{A},(3,1)}: & 0 & 0 & 0 & -1 & 1 & 0 & 1 & -1 & 0\end{array}$

Similarly we have edges $(1,3),(2,1),(2,3)$ and $(3,2)$ to be added onto the 3 arborescence $T_{B}$ in Figure 1 and edges $(1,2),(1,3),(2,1)$ and $(2,3)$ for the 3 arborescence $T_{C}$.

$\begin{array}{cccccccccc}W^{T_{B},(1,3)}: & -1 & 0 & 1 & 0 & 0 & 0 & 1 & 0 & -1 \\ W^{T_{B},(2,1)}: & -1 & 1 & 0 & 1 & -1 & 0 & 0 & 0 & 0 \\ W^{T_{B},(2,3)}: & -1 & 1 & 0 & 0 & -1 & 1 & 1 & 0 & -1 \\ W^{T_{B},(3,2)}: & 1 & -1 & 0 & 0 & 0 & 0 & -1 & 1 & 0 \\ W^{T_{C},(1,2)}: & -1 & 1 & 0 & 0 & 0 & 0 & 1 & -1 & 0 \\ W^{T_{C},(1,3)}: & -1 & 0 & 1 & 0 & 0 & 0 & 1 & 0 & -1 \\ W^{T_{C},(2,1)}: & 0 & 0 & 0 & 1 & -1 & 0 & -1 & 1 & 0 \\ W^{T_{C},(2,3)}: & 0 & 0 & 0 & 0 & -1 & 1 & 0 & 1 & -1\end{array}$

3.5 Step 4: The multivariate generating function of $C_{n}$

Because each $\bar{D}_{T}$ in the triangulation of $\bar{D}_{n}$ is unimodular, so is the dual cone $\bar{C}_{T}$ of $\bar{D}_{T}$. By Lemma 2.7, we conclude that $C_{T}$ is unimodular and that the following proposition holds:

Proposition 3.20 Fixing $\ell \in[n]$, the multivariate generating function of $C_{n}$ is given by

$$
f\left(C_{n}, \mathbf{z}\right)=\sum_{T \in \operatorname{Arb}(\ell, n)} \prod_{e \notin E(T)} \frac{1}{\left(1-\prod \mathbf{z}^{W^{T, e}}\right)} .
$$

One observes that Equation (3.4) is independent of the choice of $\ell$. Thus we have the following equality.

Corollary 3.21 For any $\ell_{1}, \ell_{2} \in[n]$,

$$
\sum_{T \in \mathbf{A r b}\left(\ell_{1}, n\right)} \prod_{e \notin E(T)} \frac{1}{\left(1-\prod \mathbf{z}^{W^{T, e}}\right)}=\sum_{T \in \operatorname{Arb}\left(\ell_{2}, n\right)} \prod_{e \notin E(T)} \frac{1}{\left(1-\prod \mathbf{z}^{W^{T, e}}\right)} .
$$




\section{A rational function formula for $f\left(t B_{n}, z\right)$}

In the last section, we obtained a formula for the multivariate generating function of the supporting cone $C_{n}$ of the vertex $I$ of $B_{n}$. Because of the symmetry of vertices of the Birkhoff polytope we can get the MFG of the supporting cone of any other vertex of $B_{n}$.

Corollary 4.1 The multivariate generating function for the lattice points of the supporting cone $C_{n}(\sigma)$ at the vertex $\sigma$, for $\sigma$ a permutation in $S_{n}$, is given by

$$
f\left(C_{n}(\sigma), \mathbf{z}\right)=\sum_{T \in \operatorname{Arb}(\ell, n)} \prod_{e \notin E(T)} \frac{1}{\left(1-\prod \mathbf{z}^{W^{T, e} \sigma}\right)},
$$

where $W^{T, e} \sigma$ is the matrix obtained from usual matrix multiplication of $W^{T, e}$ and the permutation matrix $\sigma$.

Proof [Proof of Theorem 1.1] Note that for any positive integer $t$, the supporting cone of $t B_{n}$ at vertex $t \sigma$ is still the same supporting cone $C_{n}(\sigma)$ of $B_{n}$ at the vertex $\sigma$. Then the theorem follows from Corollary 2.4 and Corollary 4.1.

We conclude this section with an example of Theorem 1.1 for our running example.

Example 4.2 When $n=3, \ell=3$, the three 3-arborescences are shown in Figure 1. In example 3.19, we have already calculated $W^{T, e}$ 's. By plugging them in, we get the three parts of the products of rational functions contributing to $f\left(C_{3}, \mathbf{z}\right)$ :

$$
\begin{aligned}
\prod_{e \notin E\left(T_{A}\right)} \frac{1}{\left(1-\prod \mathbf{z}^{W^{T_{A}, e}}\right)}= & \frac{1}{1-z_{1,2} z_{2,1} z_{1,1}^{-1} z_{2,2}^{-1}} \times \frac{1}{1-z_{1,3} z_{3,2} z_{2,1} z_{1,1}^{-1} z_{2,2}^{-1} z_{3,3}^{-1}} \\
& \times \frac{1}{1-z_{2,3} z_{3,2} z_{2,2}^{-1} z_{3,3}^{-1}} \times \frac{1}{1-z_{2,2} z_{3,1} z_{2,1}^{-1} z_{3,2}^{-1}}, \\
\prod_{e \notin E\left(T_{B}\right)} \frac{1}{\left(1-\prod \mathbf{z}^{W^{T_{B}, e}}\right)}= & \frac{1}{1-z_{1,3} z_{3,1} z_{1,1}^{-1} z_{3,3}^{-1}} \times \frac{1}{1-z_{1,2} z_{2,1} z_{1,1}^{-1} z_{2,2}^{-1}} \\
& \times \frac{1}{1-z_{2,3} z_{3,1} z_{1,2} z_{1,1}^{-1} z_{2,2}^{-1} z_{3,3}^{-1}} \times \frac{1}{1-z_{1,1} z_{3,2} z_{1,2}^{-1} z_{3,1}^{-1}},
\end{aligned}
$$

and

$$
\begin{aligned}
\prod_{e \notin E\left(T_{C}\right)} \frac{1}{\left(1-\prod \mathbf{z}^{W^{T, e}}\right)}= & \frac{1}{1-z_{1,2} z_{3,1} z_{1,1}^{-1} z_{3,2}^{-1}} \times \frac{1}{1-z_{1,3} z_{3,1} z_{1,1}^{-1} z_{3,3}^{-1}} \\
& \times \frac{1}{1-z_{2,1} z_{3,2} z_{2,2}^{-1} z_{3,1}^{-1}} \times \frac{1}{1-z_{2,3} z_{3,2} z_{3,3}^{-1} z_{2,2}^{-1}} .
\end{aligned}
$$


Thus, $\mathbf{z}^{t I} f\left(C_{3}, \mathbf{z}\right)$ equals the sum of the three rational functions multiplied by $\left(z_{1,1}^{t} z_{2,2}^{t} z_{3,3}^{t}\right)$.

In order to compute the same for other vertices we simply permute the results:

$$
\begin{aligned}
& \mathbf{z}^{t \sigma} f\left(C_{3}(\sigma), \mathbf{z}\right) \\
& =\left(z_{1, \sigma(1)}^{t} z_{2, \sigma(2)}^{t} z_{3, \sigma(3)}^{t}\right) \\
& \times\left(\frac{1}{1-z_{1, \sigma(2)} z_{2, \sigma(1)} z_{1, \sigma(1)}^{-1} z_{2, \sigma(2)}^{-1}}\right. \\
& \times \frac{1}{1-z_{1, \sigma(3)} z_{3, \sigma(2)} z_{2, \sigma(1)} z_{1, \sigma(1)}^{-1} z_{2, \sigma(2)}^{-1} z_{3, \sigma(3)}^{-1}} \\
& \times \frac{1}{1-z_{2, \sigma(3)} z_{3, \sigma(2)} z_{2, \sigma(2)}^{-1} z_{3, \sigma(3)}^{-1}} \times \frac{1}{1-z_{2, \sigma(2)} z_{3, \sigma(1)} z_{2, \sigma(1)}^{-1} z_{3, \sigma(2)}^{-1}} \\
& +\frac{1}{1-z_{1, \sigma(3)} z_{3, \sigma(1)} z_{1, \sigma(1)}^{-1} z_{3, \sigma(3)}^{-1}} \times \frac{1}{1-z_{1, \sigma(2)} z_{2, \sigma(1)} z_{1, \sigma(1)}^{-1} z_{2, \sigma(2)}^{-1}} \\
& \times \frac{1}{1-z_{2, \sigma(3)} z_{3, \sigma(1)} z_{1, \sigma(2)} z_{1, \sigma(1)}^{-1} z_{2, \sigma(2)}^{-1} z_{3, \sigma(3)}^{-1}} \\
& \times \frac{1}{1-z_{1, \sigma(1)} z_{3, \sigma(2)} z_{1, \sigma(2)}^{-1} z_{3, \sigma(1)}^{-1}} \\
& +\frac{1}{1-z_{1, \sigma(2)} z_{3, \sigma(1)} z_{1, \sigma(1)}^{-1} z_{3, \sigma(2)}^{-1}} \times \frac{1}{1-z_{1, \sigma(3)} z_{3, \sigma(1)} z_{1, \sigma(1)}^{-1} z_{3, \sigma(3)}^{-1}} \\
& \left.\times \frac{1}{1-z_{2, \sigma(1)} z_{3, \sigma(2)} z_{2, \sigma(2)}^{-1} z_{3, \sigma(1)}^{-1}} \times \frac{1}{1-z_{2, \sigma(3)} z_{3, \sigma(2)} z_{3, \sigma(3)}^{-1} z_{2, \sigma(2)}^{-1}}\right) .
\end{aligned}
$$

Finally, the summation of all six $\mathbf{z}^{t \sigma} f\left(C_{3}(\sigma), \mathbf{z}\right)$ gives $f\left(t B_{3}, \mathbf{z}\right)$.

\section{The Coefficients of the Ehrhart polynomial of the Birkhoff polytope}

In section 5.2 of [4], Barvinok and Pommersheim derive a formula for the number of lattice points of a given integral convex polytope $P$ in terms of Todd polynomial by residue computation of the MGF of $P$. When $P$ is an integral polytope, their formula explicitly indicates formulas for the coefficients of the Ehrhart polynomial $e(P, t)$ of $P$. Especially, this gives us a formula for the volume $\operatorname{vol}(P)$ of $P$, applying it we can get Theorem 1.2. We start this section by briefly recalling related results in [4].

Definition 5.1 Consider the function

$$
G\left(\tau ; \xi_{1}, \ldots, \xi_{d}\right)=\prod_{i=1}^{d} \frac{\tau \xi_{i}}{1-\exp \left(-\tau \xi_{i}\right)}
$$


in $d+1$ (complex) variables $\tau$ and $\xi_{1}, \ldots, \xi_{l}$. The function $G$ is analytic in a neighborhood of the origin $\tau=\xi_{1}=\ldots=\xi_{d}=0$ and therefore there exists an expansion

$$
G\left(\tau ; \xi_{1}, \ldots, \xi_{d}\right)=\sum_{j=0}^{+\infty} \tau^{j} \operatorname{td}_{j}\left(\left\{\xi_{i} \mid 1 \leq i \leq d\right\}\right),
$$

where $\operatorname{td}_{j}\left(\left\{\xi_{i} \mid 1 \leq i \leq d\right\}\right)=\operatorname{td}_{j}\left(\xi_{1}, \xi_{2}, \ldots, \xi_{d}\right)$ is a homogeneous polynomial of degree $j$, called the $j$-th Todd polynomial in $\xi_{1}, \ldots, \xi_{d}$. It is well-know that $\operatorname{td}_{j}\left(\left\{\xi_{i} \mid 1 \leq\right.\right.$ $i \leq d\})$ is a symmetric polynomial with rational coefficients. See page 110 in [18] for more information on Todd polynomials.

Example 5.2 Here are the first three Todd polynomials when $d=3$ :

$$
\begin{gathered}
\operatorname{td}_{3}\left(x_{1}, x_{2}, x_{3}\right)=(1 / 24)\left(x_{1}+x_{2}+x_{3}\right)\left(x_{1} x_{2}+x_{2} x_{3}+x_{3} x_{1}\right) \\
\operatorname{td}_{2}\left(x_{1}, x_{2}, x_{3}\right)=(1 / 12) x_{2}^{2}+(1 / 4) x_{3} x_{1}+(1 / 12) x_{3}^{2}+(1 / 12) x_{1}^{2}+(1 / 4) x_{2} x_{3} \\
+(1 / 4) x_{1} x_{2},
\end{gathered}
$$

$\operatorname{td}_{1}\left(x_{1}, x_{2}, x_{3}\right)=(1 / 2) x_{1}+(1 / 2) x_{2}+(1 / 2) x_{3}$, and as usual $\operatorname{td}_{0}\left(x_{1}, x_{2}, x_{3}\right)=1$.

Lemma 5.3 (See Algorithm 5.2 in [4]) Suppose $P \subset \mathbb{R}^{N}$ is a d-dimensional integral polytope and the multivariate generating function of $P$ is given by

$$
f(P, \mathbf{z})=\sum_{i} \epsilon_{i} \frac{\mathbf{z}_{i}^{a_{i}}}{\left(1-\mathbf{z}^{b_{i, 1}}\right) \cdots\left(1-\mathbf{z}^{b_{i, d}}\right)},
$$

where $\epsilon_{i}=\{-1,1\}, a_{i}, b_{i, 1}, \ldots, b_{i, d} \in \mathbb{Z}^{N}$, the $a_{i}$ 's are all vertices (with multiple occurrences) of $P$, and cone $\left(b_{i, 1}, \ldots, b_{i, d}\right)$ is unimodular, for each $i$. For any choice of $c \in \mathbb{R}^{N}$ such that $\left\langle c, b_{i, j}\right\rangle \neq 0$ for each $i$ and $j$, we have a formula for the number of lattice points in $P$ :

$$
\left|P \cap \mathbb{Z}^{N}\right|=\sum_{i} \frac{\epsilon_{i}}{\prod_{j=1}^{d}\left\langle c, b_{i, j}\right\rangle} \sum_{k=0}^{d} \frac{\left(\left\langle c, a_{i}\right\rangle\right)^{k}}{k !} \operatorname{td}_{d-k}\left(\left\langle c, b_{i, 1}\right\rangle, \ldots,\left\langle c, b_{i, d}\right\rangle\right) .
$$

Indeed, if we make the substitution $x_{i}=\exp \left(\tau c_{i}\right)$ Formula (5.1) can be rewritten as

$$
f(P, \mathbf{z})=\frac{1}{\tau^{d}} \sum_{i} \epsilon_{i} \frac{\tau^{d} \exp \left(\left\langle c, a_{i}\right\rangle\right.}{\left(1-\exp \left(\left\langle c, b_{i, 1}\right\rangle\right) \cdots\left(1-\exp \left(\left\langle c, b_{i, d}\right\rangle\right)\right.\right.} .
$$

Each fraction is a holomorphic function in a neighborhood of $\tau$ and the $d$-th coefficient of its Taylor series is a linear combination of Todd polynomials. Thus its $d$-coefficient of the Taylor series is

$$
\frac{1}{\left(\left\langle c, b_{i, 1}\right\rangle\right) \cdots\left(\left\langle c, b_{i, d}\right\rangle\right)} \sum_{k=0}^{d} \frac{\left(\left\langle c, a_{i}\right\rangle\right)^{k}}{k !} \operatorname{td}_{d-k}\left(\left\langle c, b_{i, 1}\right\rangle, \ldots,\left\langle c, b_{i, d}\right\rangle\right) .
$$


Formula (5.2) is the result of adding these contributions for each rational fraction summand.

It is clear that if Formula (5.1) is the MGF of an integral polytope $P$, then we have the MGF of any of its dilations:

$$
f(t P, \mathbf{z})=\sum_{i} \epsilon_{i} \frac{\mathbf{z}^{t a_{i}}}{\left(1-\mathbf{z}^{b_{i, 1}}\right) \cdots\left(1-\mathbf{z}^{b_{i, d}}\right)} .
$$

Hence, by using Lemma 5.3, we get the Ehrhart polynomial of $P$.

Lemma 5.4 Suppose $P \subset \mathbb{R}^{N}$ is a d-dimensional integral polytope and the multivariate generating function of $P$ (produced by Barvinok's algorithm) is given by (5.1). For any choice of $c \in \mathbb{R}^{N}$ such that $\left\langle c, b_{i, j}\right\rangle \neq 0$ for each $i$ and $j$, the Ehrhart polynomial of $P$ is

$$
e(P, t)=\sum_{k=0}^{d} \frac{t^{k}}{k !} \sum_{i} \frac{\epsilon_{i}}{\prod_{j=1}^{d}\left\langle c, b_{i, j}\right\rangle}\left(\left\langle c, a_{i}\right\rangle\right)^{k} \operatorname{td}_{d-k}\left(\left\langle c, b_{i, 1}\right\rangle, \ldots,\left\langle c, b_{i, d}\right\rangle\right) .
$$

In particular, we get a formula for the volume of $P$ :

$$
\operatorname{vol}(P)=\frac{1}{d !} \sum_{i} \epsilon_{i} \frac{\left(\left\langle c, a_{i}\right\rangle\right)^{d}}{\prod_{j=1}^{d}\left\langle c, b_{i, j}\right\rangle} .
$$

Proof Formula (5.6) follows directly from Lemma 5.3 and our earlier discussion. Formula (5.7) follows from the facts that the leading coefficient of $e(P, t)$ is $\operatorname{vol}(P)$ and the 0 -th Todd polynomial is always the constant 1 , which can be shown from the Taylor expression of the function $G\left(\tau, \xi_{1}, \ldots, \xi_{d}\right)$ defining the Todd polynomials.

Proof of Corollary 1.2 It follows from Lemma 5.4 and Theorem 1.1.

To help our readers we wrote an interactive MAPLE implementation of Formula (1.2) in the case of $B_{3}$. It is available at http://www.math.ucdavis.edu/ deloera/ RECENT_WORK/volBirkhoff3.

Clearly, it would be desirable to apply a suitable variable substitution of $c_{i, j}$ so that the expression of the volume has as few terms as possible (preferably keeping the size of $c_{i, j}$ small), with the hope of speeding up the calculations or even in the hope of finding a purely combinatorial summation. We leave this challenge to the reader and conclude with a variable exchange that gives the volume in just two variables (it is possible to leave it as a univariate rational function from the substitution $c_{i, j}=i t^{j}$ ). If we set $c_{i, j}=s^{i} t^{j}$ clearly there will be no cancellations. For example for the case $n=3$, the volume of $B_{3}$ equals.

$$
\begin{aligned}
& 1 / 24 \frac{\left(s t+s^{2} t^{2}+s^{3} t^{3}\right)^{4}}{\left(s t^{2}+s^{2} t-s t-s^{2} t^{2}\right)\left(s^{2} t^{3}+s^{3} t^{2}-s^{2} t^{2}-s^{3} t^{3}\right)\left(s t^{3}+s^{3} t^{2}+s^{2} t-s t-s^{2} t^{2}-s^{3} t^{3}\right)\left(s^{2} t^{2}+s^{3} t-s^{2} t-s^{3} t^{2}\right)} \\
& \quad+1 / 24 \frac{\left(s t+s^{2} t^{3}+s^{3} t^{2}\right)^{4}}{\left(s t^{3}+s^{2} t-s t-s^{2} t^{3}\right)\left(s^{2} t^{2}+s^{3} t^{3}-s^{2} t^{3}-s^{3} t^{2}\right)\left(s t^{2}+s^{3} t^{3}+s^{2} t-s t-s^{2} t^{3}-s^{3} t^{2}\right)\left(s^{2} t^{3}+s^{3} t-s^{2} t-s^{3} t^{3}\right)}
\end{aligned}
$$




$$
\begin{aligned}
& +1 / 24 \frac{\left(s t^{2}+s^{2} t+s^{3} t^{3}\right)^{4}}{\left(s t+s^{2} t^{2}-s t^{2}-s^{2} t\right)\left(s^{2} t^{3}+s^{3} t-s^{2} t-s^{3} t^{3}\right)\left(s t^{3}+s^{3} t+s^{2} t^{2}-s t^{2}-s^{2} t-s^{3} t^{3}\right)\left(s^{2} t+s^{3} t^{2}-s^{2} t^{2}-s^{3} t\right)} \\
& +1 / 24 \frac{\left(s t^{2}+s^{2} t^{3}+s^{3} t\right)^{4}}{\left(s t^{3}+s^{2} t^{2}-s t^{2}-s^{2} t^{3}\right)\left(s^{2} t+s^{3} t^{3}-s^{2} t^{3}-s^{3} t\right)\left(s t+s^{3} t^{3}+s^{2} t^{2}-s t^{2}-s^{2} t^{3}-s^{3} t\right)\left(s^{2} t^{3}+s^{3} t^{2}-s^{2} t^{2}-s^{3} t^{3}\right)} \\
& +1 / 24 \frac{\left(s t^{3}+s^{2} t+s^{3} t^{2}\right)^{4}}{\left(s t+s^{2} t^{3}-s t^{3}-s^{2} t\right)\left(s^{2} t^{2}+s^{3} t-s^{2} t-s^{3} t^{2}\right)\left(s t^{2}+s^{3} t+s^{2} t^{3}-s t^{3}-s^{2} t-s^{3} t^{2}\right)\left(s^{2} t+s^{3} t^{3}-s^{2} t^{3}-s^{3} t\right)} \\
& +1 / 24 \frac{\left(s t^{3}+s^{2} t^{2}+s^{3} t\right)^{4}}{\left(s t^{2}+s^{2} t^{3}-s t^{3}-s^{2} t^{2}\right)\left(s^{2} t+s^{3} t^{2}-s^{2} t^{2}-s^{3} t\right)\left(s t+s^{3} t^{2}+s^{2} t^{3}-s t^{3}-s^{2} t^{2}-s^{3} t\right)\left(s^{2} t^{2}+s^{3} t^{3}-s^{2} t^{3}-s^{3} t^{2}\right)} \\
& +1 / 24 \frac{\left(s t+s^{2} t^{2}+s^{3} t^{3}\right)^{4}}{\left(s t^{2}+s^{2} t-s t-s^{2} t^{2}\right)\left(s t^{3}+s^{3} t-s t-s^{3} t^{3}\right)\left(s^{2} t^{3}+s^{3} t+s t^{2}-s t-s^{2} t^{2}-s^{3} t^{3}\right)\left(s t+s^{3} t^{2}-s t^{2}-s^{3} t\right)} \\
& +1 / 24 \frac{\left(s t+s^{2} t^{3}+s^{3} t^{2}\right)^{4}}{\left(s t^{3}+s^{2} t-s t-s^{2} t^{3}\right)\left(s t^{2}+s^{3} t-s t-s^{3} t^{2}\right)\left(s^{2} t^{2}+s^{3} t+s t^{3}-s t-s^{2} t^{3}-s^{3} t^{2}\right)\left(s t+s^{3} t^{3}-s t^{3}-s^{3} t\right)} \\
& +1 / 24 \frac{\left(s t^{2}+s^{2} t+s^{3} t^{3}\right)^{4}}{\left(s t+s^{2} t^{2}-s t^{2}-s^{2} t\right)\left(s t^{3}+s^{3} t^{2}-s t^{2}-s^{3} t^{3}\right)\left(s^{2} t^{3}+s^{3} t^{2}+s t-s t^{2}-s^{2} t-s^{3} t^{3}\right)\left(s t^{2}+s^{3} t-s t-s^{3} t^{2}\right)} \\
& +1 / 24 \frac{\left(s t^{2}+s^{2} t^{3}+s^{3} t\right)^{4}}{\left(s t^{3}+s^{2} t^{2}-s t^{2}-s^{2} t^{3}\right)\left(s t+s^{3} t^{2}-s t^{2}-s^{3} t\right)\left(s^{2} t+s^{3} t^{2}+s t^{3}-s t^{2}-s^{2} t^{3}-s^{3} t\right)\left(s t^{2}+s^{3} t^{3}-s t^{3}-s^{3} t^{2}\right)} \\
& +1 / 24 \frac{\left(s t^{3}+s^{2} t+s^{3} t^{2}\right)^{4}}{\left(s t+s^{2} t^{3}-s t^{3}-s^{2} t\right)\left(s t^{2}+s^{3} t^{3}-s t^{3}-s^{3} t^{2}\right)\left(s^{2} t^{2}+s^{3} t^{3}+s t-s t^{3}-s^{2} t-s^{3} t^{2}\right)\left(s t^{3}+s^{3} t-s t-s^{3} t^{3}\right)} \\
& +1 / 24 \frac{\left(s t^{3}+s^{2} t^{2}+s^{3} t\right)^{4}}{\left(s t^{2}+s^{2} t^{3}-s t^{3}-s^{2} t^{2}\right)\left(s t+s^{3} t^{3}-s t^{3}-s^{3} t\right)\left(s^{2} t+s^{3} t^{3}+s t^{2}-s t^{3}-s^{2} t^{2}-s^{3} t\right)\left(s t^{3}+s^{3} t^{2}-s t^{2}-s^{3} t^{3}\right)} \\
& +1 / 24 \frac{\left(s t+s^{2} t^{2}+s^{3} t^{3}\right)^{4}}{\left(s t^{3}+s^{3} t-s t-s^{3} t^{3}\right)\left(s^{2} t^{3}+s^{3} t^{2}-s^{2} t^{2}-s^{3} t^{3}\right)\left(s t^{2}+s^{3} t-s t-s^{3} t^{2}\right)\left(s^{2} t+s^{3} t^{2}-s^{2} t^{2}-s^{3} t\right)} \\
& +1 / 24 \frac{\left(s t+s^{2} t^{3}+s^{3} t^{2}\right)^{4}}{\left(s t^{2}+s^{3} t-s t-s^{3} t^{2}\right)\left(s^{2} t^{2}+s^{3} t^{3}-s^{2} t^{3}-s^{3} t^{2}\right)\left(s t^{3}+s^{3} t-s t-s^{3} t^{3}\right)\left(s^{2} t+s^{3} t^{3}-s^{2} t^{3}-s^{3} t\right)} \\
& +1 / 24 \frac{\left(s t^{2}+s^{2} t+s^{3} t^{3}\right)^{4}}{\left(s t^{3}+s^{3} t^{2}-s t^{2}-s^{3} t^{3}\right)\left(s^{2} t^{3}+s^{3} t-s^{2} t-s^{3} t^{3}\right)\left(s t+s^{3} t^{2}-s t^{2}-s^{3} t\right)\left(s^{2} t^{2}+s^{3} t-s^{2} t-s^{3} t^{2}\right)} \\
& +1 / 24 \frac{\left(s t^{2}+s^{2} t^{3}+s^{3} t\right)^{4}}{\left(s t+s^{3} t^{2}-s t^{2}-s^{3} t\right)\left(s^{2} t+s^{3} t^{3}-s^{2} t^{3}-s^{3} t\right)\left(s t^{3}+s^{3} t^{2}-s t^{2}-s^{3} t^{3}\right)\left(s^{2} t^{2}+s^{3} t^{3}-s^{2} t^{3}-s^{3} t^{2}\right)} \\
& +1 / 24 \frac{\left(s t^{3}+s^{2} t+s^{3} t^{2}\right)^{4}}{\left(s t^{2}+s^{3} t^{3}-s t^{3}-s^{3} t^{2}\right)\left(s^{2} t^{2}+s^{3} t-s^{2} t-s^{3} t^{2}\right)\left(s t+s^{3} t^{3}-s t^{3}-s^{3} t\right)\left(s^{2} t^{3}+s^{3} t-s^{2} t-s^{3} t^{3}\right)} \\
& +1 / 24 \frac{\left(s t^{3}+s^{2} t^{2}+s^{3} t\right)^{4}}{\left(s t+s^{3} t^{3}-s t^{3}-s^{3} t\right)\left(s^{2} t+s^{3} t^{2}-s^{2} t^{2}-s^{3} t\right)\left(s t^{2}+s^{3} t^{3}-s t^{3}-s^{3} t^{2}\right)\left(s^{2} t^{3}+s^{3} t^{2}-s^{2} t^{2}-s^{3} t^{3}\right)} \text {. }
\end{aligned}
$$

\section{Integration of polynomials and volumes of faces of the Birkhoff polytope}

In this final section we look at two more applications of Theorem 1.1, going beyond the computation of Ehrhart coefficients.

The first application is to the integration of polynomials over $B_{n}$. The main observation is that, once we know a unimodular cone decomposition for the supporting cones at all vertices of $B_{n}$, a formula for the integral (see Formula (6.1)) follows from Brion's theorem on polyhedra $[2,8]$.

Theorem 6.1 Suppose $P \subset \mathbb{R}^{N}$ is a d-dimensional integral polytope and the multivariate generating function of $P$ is given precisely by Formula (5.1), i.e., we have full 
knowledge of a unimodular cone decomposition for each of $P$ 's supporting cones and its rays $b_{i, j}$ and vertices $a_{i}$. Then for any choice of $y \in \mathbb{R}^{N}$ such that $\left\langle y, b_{i, j}\right\rangle \neq 0$ for each $i$ and $j$, we get a formula for the integral the $p$-th power of a linear form over $P$

$$
\int_{P}\langle y, x\rangle^{p} d x=\frac{(-1)^{d}}{(p+1)(p+2) \ldots(p+d)} \sum_{i} \epsilon_{i} \frac{\left(\left\langle y, a_{i}\right\rangle\right)^{p+d}}{\prod_{j=1}^{d}\left\langle y, b_{i, j}\right\rangle} .
$$

Notice that although each term in the sum has poles, the poles cancel and the sum is an analytic function of $y$. Since the $p$ th-powers of linear forms generate the whole vector spaces of polynomials, one obtains, from Theorem 1.1, a formula of integration for the polynomial functions over $B_{n}$ (or for that matter, for any integral polytope for which we understand its cone decomposition).

The next application is to the computation of Ehrhart polynomials of faces of $B_{n}$. One can easily obtain from Theorem 1.1 similar formulas for the nonnegative integral semi-magic squares with structural zeros or forbidden entries (i.e. fixed entries are equal zero). Note that any face $F$ of $B_{n}$, being the intersection of finitely many facets, is determined uniquely by the set of entries forced to take the value zero. To obtain a generating function for the dilations of a face $F$ of $B_{n}, f(t F, \mathbf{z})$, we start from our formula for $f\left(t B_{n}, z\right)$ in Theorem 1.1. For those variables $x_{i j}$ mandated to be zero, we select a vector $\lambda$ with entries $\lambda_{i j} \geq 0$ so that the substitution $x_{i j}:=s^{\lambda_{i j}}$ does not create a singularity (this $\lambda$ exists e.g., by taking random values from the positive orthant). Call $g(t F, \mathbf{z}, s)$ the result of doing this substitution on $f\left(t B_{n}, \mathbf{z}\right)$. We will eventually set $s=0$, but first, let us check that this will give no singularities. $s$ can only appear in the numerator with a nonnegative exponent. It can potentially create a singularity if it appears in a factor of the denominator with negative exponent. But, if this occurs, $s$ can be factored out and put with a positive exponent in the numerator. Thus, we can safely resolve the singularity. Now, we set $s=0$ in $g(t F, \mathbf{z}, s)$, those terms that had a power of $s$ in the numerator disappear. We obtain a multivariate sum of rational functions that gives us only the desired lattice points inside $t F$. We have now two examples of this method. First we apply it to obtain a table with the Ehrhart polynomials for a (any) facet of $B_{3}, B_{4}, B_{5}, B_{6}$.

\begin{tabular}{ll}
\hline $\mathrm{n}$ & Ehrhart polynomial of a facet of $B_{n}$ \\
\hline 3 & $1+\frac{11}{6} t+t^{2}+\frac{1}{6} t^{3}$ \\
1 & $+\frac{471}{140} t+\frac{1594}{315} t^{2}+\frac{73}{16} t^{3}+\frac{161}{60} t^{4}+\frac{83}{80} t^{5}+\frac{61}{240} t^{6}+\frac{1}{28} t^{7}+\frac{11}{5040} t^{8}$ \\
1 & $+\frac{1752847}{360360} t+\frac{904325}{77616} t^{2}+\frac{147579347}{8072064} t^{3}+\frac{8635681}{415800} t^{4}+\frac{6412937357}{359251200} t^{5}+\frac{18455639}{1555200} t^{6}$ \\
& $+\frac{1611167963}{261273600} t^{7}+\frac{95702009}{38102400} t^{8}+\frac{365214839}{457228800} t^{9}+\frac{5561}{28350} t^{10}+\frac{52388227}{1437004800} t^{11}+\frac{42397}{8553600} t^{12}$ \\
& $+\frac{4342517}{9340531200} t^{13}+\frac{22531}{838252800} t^{14}+\frac{188723}{261534873600} t^{15}$ \\
1 & $+\frac{87450005}{13728792} t+\frac{102959133218657}{4947307485120} t^{2}+\frac{14843359499161}{322075353600} t^{3}+\frac{230620928072832499}{3011404556160000} t^{4}+\frac{237290485580450429}{2365321396838400} t^{5}$ \\
& $+\frac{15435462135033037}{144815595724800} t^{6}+\frac{108878694347719}{1164067946496} t^{7}+\frac{439368248888657369}{6402373705728000} t^{8}+\frac{447656681773591807}{1054508610355200} t^{9}$ \\
& $+\frac{434798171323757}{19527937228800} t^{10}+\frac{1047553900202141}{105450861035520} t^{11}+\frac{250284934507924171}{66283398365184000} t^{12}+\frac{28330897394929}{23176013414400} t^{13}$ \\
& $+\frac{2229552439625171}{6628339836518400} t^{14}+\frac{6610306048279}{84360688828416} t^{15}+\frac{215934508972451}{14060114804736000} t^{16}+\frac{2045239925737}{814847562547200} t^{17}$ \\
& $+\frac{1729908621731}{5121898964582400} t^{18}+\frac{21042914689}{572447531335680} t^{19}+\frac{6138921521069}{1946321606541312000} t^{20}+\frac{1398566666897}{681212562289459200} t^{21}$ \\
& $+\frac{47580345877}{4995558790122700800} t^{22}+\frac{4394656999}{15667888932657561600} t^{23}+\frac{9700106723}{24662096832274759680000} t^{24}$
\end{tabular}


With the same method we have also computed, for the first time, the Ehrhart polynomials for the Chan-Robbins-Yuen polytopes $C R Y_{3}, C R Y_{4}, C R Y_{5}, C R Y_{6}$, and $\mathrm{CRY}_{7}$ (see [12]):

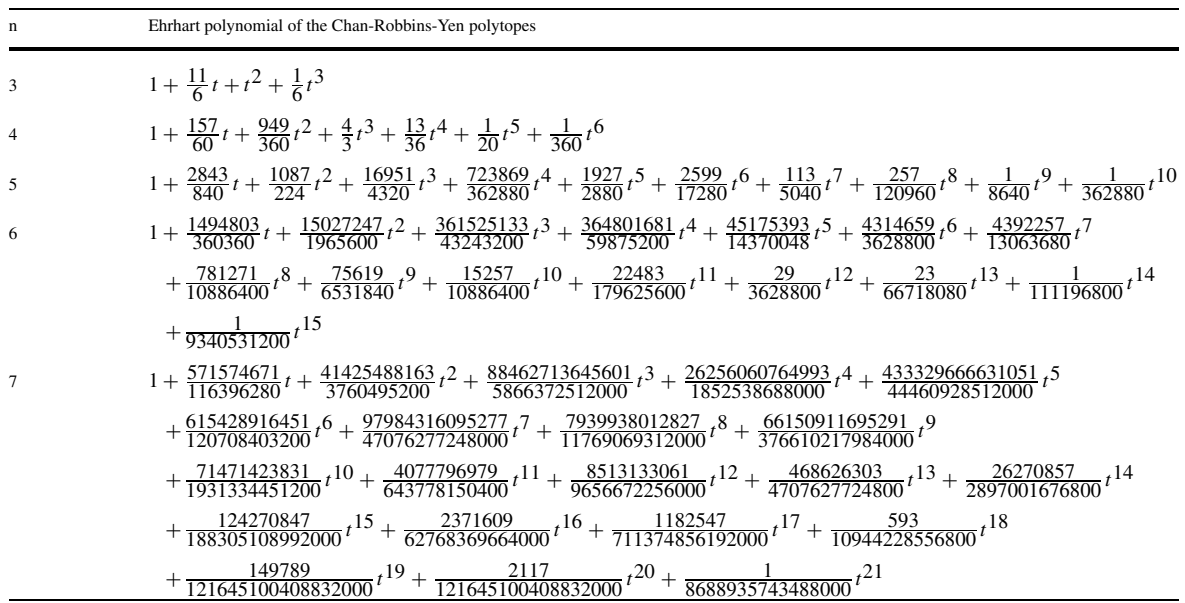

We conclude with some remarks. First, it is natural to ask whether one can derive our volume formula from a perturbation of the Birkhoff polytope and then applying Lawrence's formula for simple polytopes [20]. We found such a proof using a perturbation suggested by B. Sturmfels, but the proof presented here yields more results, for example, Corollaries 3.21 and 4.1 can only be obtained this way. Second, it is well known that Brion's and Lawrence's formulas can be proved from the properties of characteristic functions of polyhedra under polarity (see e.g. Corollary 2.8 in [4] or Theorem 3.2 [6]). On the other hand P. Filliman [17, 19] expressed the characteristic function of any convex polytope $P$ containing the origin as an alternating sum of simplices that share supporting hyperplanes with $P$. The terms in the alternating sum are given by a triangulation of the polar polytope of $P$. Filliman's machinery yields in a limiting case Lawrence's volume formulas. Different choices of triangulation of the polar of $P$ yield different volume formulas for $P$. Using Filliman's duality G. Kuperberg found (unpublished) other special volume formulas that follow from pulling triangulations of the dual of $B_{n}$.

Acknowledgements We are truly grateful to Richard P. Stanley for his encouragement and support. In fact it is because of Prof. Stanley that this project got started, after he alerted us that there was a really nice pattern in the data presented in Table 2 of [13]. We are also grateful to Bernd Sturmfels and Günter Ziegler for useful conversations that eventually led us to discover the combinatorial statement proved in Theorem 1.1. We have also received useful suggestions and references from Alexander Barvinok, Matthias Beck, Greg Kuperberg, Alexander Postnikov, and Peter Huggins.

\section{References}

1. Baldoni, V., De Loera, J.A, Vergne, M.: Counting integer flows in networks. Foundations of Computational Mathematics 4(3), 277-314 (2004)

2. Barvinok, A.I.: Computing the volume, counting integral points, and exponential sums. Discrete Comput. Geom. 10, 123-141 (1993) 
3. Barvinok, A.I.: A course in convexity. Graduate studies in Mathematics, vol. 54. American Math. Soc., Providence (2002)

4. Barvinok, A.I., Pommersheim, J.: An algorithmic theory of lattice points in polyhedra. In: New Perspectives in Algebraic Combinatorics (Berkeley, CA, 1996-1997). Math. Sci. Res. Inst. Publ., vol. 38, pp. 91-147. Cambridge Univ. Press, Cambridge (1999)

5. Beck, M., Pixton, D.: The Ehrhart polynomial of the Birkhoff polytope. Discrete Comput. Geom. 30, 623-637 (2003)

6. Beck, M., Hasse, C., Sottile, F.: Theorems of Brion, Lawrence, and Varchenko on rational generating functions for cones, manuscript (2007), available at math ArXiv:math.CO/0506466

7. Beck, M., Robins, S.: Computing the continuous discretely: integer-point enumeration in polyhedra. Springer undergraduate texts in Mathematics (2007)

8. Brion, M.: Points entiers dans les polyèdres convexes. Annales scientifiques de l'École Normale Supérieure Ser. 4(21), 653-663 (1988)

9. Canfield, E.R., McKay, B.: Asymptotic enumeration of integer matrices with constant row and column sums, available at math ArXiv:CO/0703600

10. Canfield, E.R., McKay, B.: The asymptotic volume of the Birkhoff polytope, available at math ArXiv:CO/0705.2422

11. Chan, C.S., Robbins, D.P.: On the volume of the polytope of doubly-stochastic matrices. Experiment. Math. 8(3), 291-300 (1999)

12. Chan, D.P., Robbins, C.S, Yuen, D.S: On the volume of a certain polytope. Experiment. Math. 9(1), 91-99 (2000)

13. De Loera, J.A., Hemmecke, R., Tauzer, J., Yoshida, R.: Effective Lattice Point Counting in Rational Convex Polytopes. Journal of Symbolic Computation 38, 1273-1302 (2004)

14. De Loera, J.A, Rambau, J., Santos, F.: Triangulations: Structures and Algorithms. Manuscript (2008)

15. Diaconis, P., Gangolli, A.: Rectangular Arrays with Fixed Margins. IMA Series on Volumes in Mathematics and its Applications, vol. 72, pp. 15-41. Springer, Berlin (1995)

16. Ehrhart, E.: Polynômes Arithmétiques et Méthode des Polyédres en Combinatoire. Birkhauser, Basel (1977)

17. Filliman, P.: The volume of duals and sections of polytopes. Mathematika 39, 67-80 (1992)

18. Fulton, W.: Introduction to Toric Varieties. Princeton University Press, Princeton (1993). 180 pages

19. Kuperberg, G.: A generalization of Filliman duality. Proceedings of the AMS 131(12), 3893-3899 (2003)

20. Lawrence, J.: Polytope volume computation. Math. Comput. 57, 259-271 (1991)

21. Schrijver, A.: Theory of Linear and Integer Programming. Wiley, New York (1986)

22. Stanley, R.P.: Enumerative Combinatorics, 2nd ed., vol. I. Cambridge University Press, Cambridge (1997)

23. Sturmfels, B.: Gröbner Bases and Convex Polytopes. University Lecture Series, vol. 8. AMS, Providence (1995)

24. Yemelichev, V.A., Kovalev, M.M., Kratsov, M.K.: Polytopes, Graphs and Optimisation. Cambridge Univ. Press, Cambridge (1984)

25. Zeilberger, D.: Proof of a conjecture of Chan, Robbins, and Yuen. Electronic Transactions on Numerical Analysis 9, 147-148 (1999)

26. Ziegler, G.M.: Lectures on Polytopes. Graduate Texts in Mathematics, vol. 152. Springer, New York (1995). 370 pages 